\title{
SARS-CoV-2 infection leads to cardiac pericyte loss, fibrosis, cardiomyocyte hypertrophy, and diastolic dysfunction
}

\author{
Margo Daems \\ KU Leuven \\ Rega institute \\ Ilona Cuijpers \\ KU Leuven
}

Laurens Liesenborghs

\section{Robbert Boudewijns}

University of Leuven https://orcid.org/0000-0002-5984-7279

Jana Raman

KU Leuven

\section{Steven Simmonds}

KU Leuven

Nadèche Geuens

KU Leuven

Marleen Lox

KU Leuven

Peter Verhamme

KU Leuven

Sophie Van Linthout

BCRT

\section{Stephane Heymans}

Maastricht University, CARIM School for Cardiovascular Diseases

\section{Carsten Tschoepe}

Charité-Universitätsmedizin Berlin, Campus Benjamin Franklin

Johan Neyts

Rega Institute, KU Leuven https://orcid.org/0000-0002-0033-7514

Elizabeth Jones ( $\square$ liz.jones@kuleuven.be)

KU Leuven https://orcid.org/0000-0002-2006-7064 
Keywords: COVID-19, SARS-CoV-2, cardiac pericyte loss, diastolic dysfunction, cardiomyocyte hypertrophy, fibrosis

Posted Date: November 30th, 2020

DOI: https://doi.org/10.21203/rs.3.rs-105963/v1

License: (c) (1) This work is licensed under a Creative Commons Attribution 4.0 International License. Read Full License 


\section{SARS-CoV-2 infection leads to cardiac pericyte loss, fibrosis, cardiomyocyte hypertrophy, and diastolic dysfunction}

Margo Daems ${ }^{a}$, Laurens Liesenborghs ${ }^{\mathrm{a}, \mathrm{b}}$, Ilona Cuijpers ${ }^{\mathrm{a}, \mathrm{c}}$, Robbert Boudewijns ${ }^{\mathrm{a}, \mathrm{b}}$, Jana Raman ${ }^{a}$, Steven J. Simmonds ${ }^{a}$, Nadeche Geuens ${ }^{a}$, Marleen Lox ${ }^{a}$, Peter Verhamme ${ }^{a}$, Sophie Van Linthout ${ }^{d, e}$, Stephane Heymans ${ }^{\mathrm{a}, \mathrm{c}, \mathrm{f}}$, Cartsen Tschöpe ${ }^{\mathrm{d}, \mathrm{e}, \mathrm{g}}$, Johan Neyts ${ }^{\mathrm{b}}$, Elizabeth A.V. Jones $^{\mathrm{a}, \mathrm{c}} *$

a Centre for Molecular and Vascular Biology, KU Leuven, Herestraat 49, bus 911, 3000 Leuven, Belgium

b Laboratory of Virology and Chemotherapy, Department of Microbiology, Immunology and Transplantation, Rega Institute for Medical Research, KU Leuven Herestraat 49, bus 911, 3000 Leuven, Belgium

' Department of Cardiology, Maastricht University, CARIM School for Cardiovascular Diseases, Universiteitssingel 50, 6229 ER Maastricht, Netherlands

${ }^{d}$ Berlin Institute of Health Center for Regenerative Therapies (BCRT) Charité - University Medicine Berlin Campus Virchow Clinic Berlin Germany.

e German Center for Cardiovascular Research (DZHK), partner site Berlin, Germany ${ }^{f}$ ICIN-Netherlands Heart Institute, Holland Heart House, Moreelsepark 1, 3511 EP Utrecht, Netherlands

g Department of Cardiology and Pneumology, Charité, University Medicine Berlin, Campus Virchow Klinikum, Berlin, Germany

Running title: COVID19 induced heart failure Corresponding author:

Elizabeth A V Jones

Center for Molecular and Vascular Biology, KU Leuven, Herestraat 49, bus 911, 3000 Leuven, Belgium E-mail: liz.jones@kuleuven.be 


\section{ABSTRACT}

Recovered COVID19 patients often display cardiac dysfunction, even after a relatively mild infection. Here, we present the first histological description of cardiac SARS-CoV-2 infection. Within the heart, the ACE2 receptor is mostly expressed by pericytes. Using a COVID19 hamster model, we demonstrate SARS-CoV-2 is replicating in pericytes, and reduced pericyte density is present after infection. In healthy animals, pericytes recover; however, when metabolic comorbidities are present, they fail to recover. These latter animals present with cardiac fibrosis, cardiomyocyte hypertrophy, and early signs of diastolic dysfunction, resembling HFpEF. Biopsies from recovered COVID19 patients showed similar results, with pericyte loss being present. 
With the number of COVID-19 cases increasing every day, the long-term consequences of SARS-CoV-2 infection, both after clinically mild or more severe manifestations, are not yet understood. SARS-CoV-2 infection has several implications beyond the traditional respiratory syndrome COVID19, including the risk of developing severe cardiac injury, which in turn may exacerbate its severity ${ }^{1-3}$. Over $60 \%$ of patients hospitalized for COVID19 present with clear signs of myocardial injury ${ }^{4}$, with between 20 and $63 \%$ of patients showing increased biomarker levels, depending on the study ${ }^{2,4,5}$. Hospitalized patients present with a range of cardiac dysfunctions, including left ventricular wall motion abnormalities $(23.7 \%)$, ventricular dysfunction (RV 26.3\%, LV18.4\%), diastolic dysfunction (13.2\%), and pericardial effusions (7.2\%) ${ }^{4}$. Additionally, long term consequences on cardiac function are suggested, as evidenced by the presence of cardiac fibrosis ${ }^{6,7}$.

The underlying mechanism by which SARS-CoV-2 infection causes cardiac injury is poorly understood. Cardiac damage could occur indirectly, due to thrombotic events or hyperinflammation targeted against the heart, or directly through viral infection of the heart ${ }^{8}$. In autopsy specimens from patients who died from COVID19, significant amounts of viral RNA were found in the heart ${ }^{9}$. Similarly, in a SARS-CoV-2-infected hamster model, the heart is among the tissues with the highest viral loads, after the respiratory tract ${ }^{10}$. Internalization of the virus depends on its binding to the angiotensin converting enzyme 2 (ACE2) receptor. In the human heart, ACE2 is mostly expressed by pericytes, and to a lesser extent by cardiomyocytes ${ }^{11,12}$. As pericytes are crucial for the microvascular function and integrity, SARS-CoV-2 infection may induce capillary dysfunction indirectly by attacking pericytes. The combination of microvascular dysfunction, inflammation, and the presence of 
post-infective cardiac fibrosis highlights the potential of developing diastolic dysfunction after recovering from COVID19. In fact, most patients with COVID19 that suffer cardiac injury present with preserved ejection fraction, but show either right ventricular dilatation (29\%) or left ventricular diastolic dysfunction (16\%) ${ }^{13}$. The former is likely indirectly caused by effects on the lung, whereas the ventricular dysfunction could be a direct effect of the cardiac SARS-CoV-2 infection itself. As such, COVID19 may be an independent risk factor for the development of heart failure with preserved ejection fraction (HFpEF) ${ }^{14}$.

Here, we use a SARS-CoV-2-infected hamster model to investigate the cardiac effects of COVID19. A reduced cardiac pericyte coverage is present very early after infection. Additionally, we show that in vitro ACE2 internalization renders pericytes more prone to vasodilatation. Finally, we demonstrate that in the presence of metabolic comorbidities (obesity, hyperlipidemia, and hypertension), SARS-CoV-2-infected hamsters fail to recover their reduced cardiac pericyte coverage and present with fibrosis and cardiomyocyte hypertrophy. Using echocardiography, we demonstrate that in the presence metabolic comorbidities, SARS-CoV-2 infection accelerate the development of diastolic dysfunction. To confirm the relevance for COVID19 patients, we investigated microvascular structure in biopsies from recovered COVID19 patients who had developed cardiac complications. All patients showed reduced pericyte coverage of capillaries, as indicated by $\alpha \mathrm{SMA}^{\text {low }}$ expression. 


\section{RESULTS}

\section{SARS-CoV-2 infects cardiac pericytes and causes a reduction in cardiac pericyte coverage}

in hamsters

To study the effect of SARS-CoV-2 infection on cardiac function, we used a previously described COVID19 hamster model ${ }^{15,16}$. In this model, hamsters are inoculated intranasally with SARS-CoV-2 virus $\left(2 \times 10^{6} \mathrm{TCID}_{50}\right)$, which leads to a peak in lung viral load at 4 days post infection (dpi). To determine whether SARS-CoV-2 infects the heart tissue, we quantified the viral load in the heart, relative to lung and muscle tissue, by using qPCR at $4 \mathrm{dpi}$. In the heart, the viral load reached a median of $\sim 4 \log _{10}$ RNA copies per mg tissue, around $3 \log _{10}$ less compared to the lung, while in the muscle only minimal amounts of viral RNA was detected (Fig. 1a). Titrations of homogenized cardiac tissue confirmed the presence of infectious particles in the heart of around $2 \log _{10} T C I D_{50} / m g$ tissue (Supplemental Fig. 1). We next investigated the expression of viral RNA in the heart using in situ hybridization at 4 dpi. Our in situ hybridization demonstrates that the SARS-CoV-2 virus was present in cells adjacent to capillaries. This indicates that SARS-CoV-2 replication occurs primarily in the pericytes surrounding the capillaries (Fig. 1b).

Given the presence of virus in the pericytes, we next investigated cardiac microvascular changes in the infected hamster hearts at $4 \mathrm{dpi}$. No difference in cardiac arteriolar density was observed, as defined by high aSMA expression (Supplemental Fig. 2). In order to identify all cardiac pericyte subsets, we used both PDGFR- $\beta$ and NG2 as markers. Infected hamsters showed a significantly reduction in the percentage of capillaries with $\mathrm{NG2}^{+}$ 
pericytes (Fig. 1C-d), while there was no difference observed in PDGFR- $\beta^{+}$pericytes (Fig. 1cd). Double-positive NG2/PDGFR- $\beta$ vessels showed a trend $(p=0.0542)$ towards a reduced pericyte density in the heart (Fig. 1c-d). These changes in cardiac pericyte coverage were not associated with any changes in vascular density at 4 dpi (Fig. 1c-d).

\section{Pericytes in vitro are more susceptible to pro-relaxation cues after ACE2 internalization}

As SARS-CoV-2 infection is associated with cardiac pericyte loss in hamsters, we questioned whether it would affect pericyte functioning as well. As such, we simulated SARS-CoV-2 infection by exposing human immortalized pericytes in vitro to recombinant SARS-CoV-2 spike protein (Fig. 2). The SARS-CoV-2 spike protein causes an internalization of ACE2, resulting in a reduced ACE2 surface expression ${ }^{17}$. Exposure of human immortalized pericytes with SARS-CoV-2 spike protein did not induce apoptosis (Fig. 2a, b), but significantly affected pericyte function. We analyzed the expression of genes involved in pericyte contraction and relaxation (Fig. 2c-f) and their interaction with endothelial cells (Supplemental Fig. 3). We found a two-fold and three-fold increase in gene expression of adenosine $\mathrm{A} 1$ receptor and adenosine $\mathrm{A} 2 \mathrm{~A}$ receptor, respectively (encoded by ADORA1 and ADORA2, Fig. 2 c-d). These genes are involved in adenosine-induced pericyte relaxation ${ }^{18}$. Similarly, transcript levels of prostaglandin E2 receptor 4 (PTGER4), a receptor involved in prostaglandin-induced pericyte relaxation, were upregulated upon SARS-CoV-2 spike protein exposure (Fig. 2e) ${ }^{19}$. We observed no change in the Adrenoceptor Alpha $2 \mathrm{~A}$ receptor, encoded by $A D R A 2 A$, which induces noradrenaline-induced pericyte contraction

(Fig. 2f) ${ }^{19,20}$. In contrast to the strong effects on genes involved in pericyte relaxation, most of the growth factors produced by pericytes sustaining endothelial cell function were only 
slightly affected by exposure to the SARS-CoV-2 spike protein, with the exception of Heparin-binding EGF-like growth factor (HBEGF), which showed an almost 2-fold increase (Supplemental Fig. 3). These results show that ACE2 internalization as a consequence of SARS-CoV-2 spike protein exposure promotes growth factor production by pericytes slightly, but makes them more sensitive to relaxation cues, possibly causing a dysregulation in microvascular flow patterns.

\section{SARS-CoV-2 infected hamsters do not recover their cardiac pericyte coverage when exposed to metabolic comorbidities}

Though our results indicate that cardiac pericytes were lost, and possibly dysfunctional, after SARS-CoV-2 infection, it was unclear whether pericytes could recover post-infection. Pericytes are also lost in other pathologies, notably diabetes ${ }^{21}$. Here, insulin treatment can partly rescue the reduced pericyte density observed ${ }^{22}$. Thus, pericytes have at least some regenerative capacities. As such, we investigated whether the heart could also recover after SARS-CoV-2 infection (Fig. 3). Since the presence of metabolic comorbidities is associated with both HFpEF and a more severe outcome after COVID19 23,24 , we investigated whether hyperlipidemia and hypertension affects the cardiac phenotype after COVID19 recovery. To this end, infected hamsters and uninfected controls received either high fat diet (HFD) combined with hypertension-inducing NY-Nitro-L-arginine methyl ester (L-NAME) or a regular chow diet ${ }^{25}$. Hamsters were put on HFD/L-NAME one week before infection (Fig. 3a). 
At $14 \mathrm{dpi}$, viral RNA was still detected in the lungs, but had decreased substantially compared to levels present at 4 dpi (Fig. 3b). In the heart, no viral RNA was detected at 14 dpi. Uninfected hamsters on HFD/L-NAME gained weight after being three weeks on diet, while the weight of SARS-CoV-2 infected animals remained stable (Fig. 3c-d).

In uninfected chow-fed hamsters, approximately $80 \%$ of cardiac capillaries are covered by pericytes expressing either PDGFR- $\beta$ and/or NG2 at 14 dpi (Fig. 3e-i). Both HFD/L-NAMEtreated uninfected hamsters and SARS-CoV-2-infected hamsters on a chow diet showed a slight but unsignificant reduction in pericyte coverage, compared to uninfected chow-fed animals (Fig 3e-f). Infected animals on HFD/L-NAME, however, presented with a reduced $\mathrm{NG}^{+}$pericyte density compared to chow-fed hamsters (Fig. 3e-i). Approximately $60 \%$ of vessels were covered with pericytes, which is similar to the observed level of pericyte coverage of infected hamsters at $4 \mathrm{dpi}$ (Fig. 1c,d,f). Overall, our results indicate that cardiac pericyte coverage does recover, but is prevented by metabolic comorbidities. When we investigated $\alpha \mathrm{SMA}$ coverage and microvascular density, no differences were observed between the groups (Supplemental Fig. 4).

Cardiac damage remaining post-infection could be indicative of future cardiac dysfunction. We therefore investigated cardiomyocyte hypertrophy and cardiac fibrosis in hamster hearts at $14 \mathrm{dpi}$ (Fig. 4). Cardiomyocytes from SARS-Cov-2 infected hearts were 20\% larger compared to cardiomyocytes from uninfected hamster hearts (Fig. 4a-b). This is the a slightly smaller range of hypertrophy reported for animal HFpEF models, where increases between $35 \%$ and $45 \%$ have been reported ${ }^{25,26}$. Other viral myocarditis models, such as CVB3 infection in mice, show approximately $15 \%$ increases in cardiomyocyte size after five weeks ${ }^{27}$, further highlighting the unique nature of this cardiac hypertrophy. We found a 
significant increase in fibrosis, both due to HFD/L-NAME, as well as infection with SARS-CoV2 (Figure 4c-d). These effects were not cumulative, as infected hamsters on treatment showed the same level of fibrosis as hamsters on treatment or infected alone.

Lastly, we investigated cardiac function at $14 \mathrm{dpi}$ by echocardiography. At this stage, few defects in cardiac function were present (Fig. 5). No differences in heart rate (HR) were observed between the groups (Fig. 5a). Systolic function was assessed by measuring ejection fraction (EF), fractional shortening (FS), and isovolumic contraction time (IVCT) (Fig. 5b, Supplemental Table S1). No difference in FS was observed (Fig. 5b). IVCT was increased in chow diet-fed infected hamsters compared to non-infected hamsters (Supplemental Table S1). Cardiac hypertrophy was also suggested by the echocardiography, with an increased LV mass present in both infected hamster groups, although statistically insignificant (Fig. 5c). Some evidence of the onset of diastolic dysfunction could be observed in the infected HFD/L-NAME hamsters. Although the E-wave increases drastically in severe, late-stage diastolic dysfunction, it decreases early in diastolic dysfunction. Here, a small but statistically significant decrease in the E-wave was present in HFD/L-NAME-treated infected hamsters compared to control uninfected hamsters (Fig. 5d). Similarly, the IVRT was slightly increased (Fig. 5e). However, the most common indicators of diastolic dysfunction, E/E', E/A, and mitral valve deceleration time (MV DT), were unaffected in all groups (Fig. $5 f-g$, Supplemental Table S1). Complete echocardiographic analysis is available in Supplemental Table S1. Together with the histological data, our echocardiography data suggest that the infected hamster exposed to comorbidities are showing early signs of diastolic dysfunction. 


\section{Recovered COVID19 patients present with reduced number of $\alpha$ SMA ${ }^{\text {low }}$ pericytes.}

Lastly, we investigated whether similar effects were observed in patients that recovered from COVID19 (Fig. 6). Biopsies were obtained from patients that developed cardiac complications, warranting catheterization. We limited our analysis to patients who presented with cardiac injury but had a preserved ejection fraction. As control tissue, we used biopsies from patients who had been suspected of developing viral myocarditis but tested negative, as well as patients diagnosed with HFpEF. We found no differences in cardiac microvascular density between control patients, HFpEF patients, and recovered COVID19 patients, based on CD34 endothelial staining (Fig. 6a-b). Human cardiac pericytes express several markers, including platelet-derived growth factor receptor beta (PDGFR- $\beta$ ), neuron-glial antigen 2 (NG2), as well as low alpha smooth muscle actin ( $\alpha \mathrm{SMA}$ ) levels ${ }^{28}$. However, not all pericytes express all these markers, suggesting that each marker may stain a different subset of pericytes. None of the tested PDGRF- $\beta$ antibodies worked on our samples, possibly due to the fixation protocol necessary for working with infected cardiac biopsies. We found no statistical difference in the percentage of $\mathrm{NG2}^{+}$capillaries for any of the patient groups, even the HFpEF patients. The data from recovered COVID19 patients was extremely variable (Supplemental Fig. 5). However, we found a significant reduction in the percentage of $\alpha \mathrm{SMA}^{\text {low }}$ capillaries in both HFpEF and COVID19 recovered patients (Fig. 6a-c). Together, these data demonstrate that both recovered COVID19 patients and HFpEF patients lose the same amount of $\alpha S_{M} A^{\text {low }}$ pericytes, independently of SARS-CoV-2 infection, suggesting that pericytes are indeed targeted by SARS-CoV-2 infection.

Though cardiomyocyte expression of ACE2 is low, several reports show that the expression increases in patients with certain comorbidities ${ }^{29}$. We therefore investigated whether 
COVID19 affects cardiomyocytes by measuring cardiomyocyte hypertrophy, but could not detect any difference in cardiomyocyte size between either patient group (Supplemental Fig. 6). We cannot, however, say with certainty that our control patients lacked hypertrophy since they presented with viral myocarditis-like symptoms.

\section{DISCUSSION}

Our results demonstrate that SARS-CoV-2 infection profoundly affects the cardiac microvascular structures by reducing pericyte coverage. This can predispose SARS-CoV-2 infected individuals to developing diastolic heart failure, especially in the presence of metabolic comorbidities. Previous reports stated that ACE2 was largely expressed by cardiac pericytes and to a lesser extent in cardiomyocytes ${ }^{11,12}$. Our in situ hybridization shows that pericytes have the highest levels of viral replication in the heart. This does not mean that the virus is not present in cardiomyocytes, but only that the virus replicates at a much higher level in pericytes.

COVID19 can in some cases lead to viral myocarditis-like syndromes ${ }^{30}$ and recovered patients show clinical indicators of cardiac injury ${ }^{31}$. Because the infection targets pericytes, our results support the idea that recovered patients are at risk of developing HFpEF. Our group has been investigating HFpEF development in the ZSF1 rodent model (Cuijpers, et al., in preparation). Our unpublished findings show that pericyte loss is the earliest parameter to change histologically and inducing pericyte loss induces HFpEF. Infected hamsters, especially those exposed to metabolic comorbidities, show histological signs of HFpEF. They have increased interstitial stiffness (fibrosis) and cardiac microvascular dysfunction, as 
shown by the loss of cardiac pericytes. At the stages investigated, diastolic dysfunction could not yet be detected, as assessed by changes in $E / E^{\prime}$ and $E / A$. However, some measures of diastolic dysfunction, specifically IVRT and the E wave velocity, began to indicate dysfunction. Therefore, our results demonstrate that COVID19 recovered patients with metabolic comorbidities should be followed closely to investigate diastolic dysfunction.

The level of pericyte loss observed in our hamster model was much milder compared to the recovered COVID19 patients (around 30\% reduction when using $\alpha$ SMA $A^{\text {low }}$ as a marker) and our animal HFpEF models (41\% pericyte loss after 14 weeks in ZSF1 rat model, Cuijpers, et al., in preparation). Pericyte loss in patients was also marker specific, possibly indicated that a specific subset of cardiac pericytes is targeted. Only hamsters with pre-existing metabolic comorbidities exhibited a difference in all pathophysiological markers (hypertrophy, fibrosis, and pericyte loss) and showed some level of diastolic dysfunction by echocardiography. Therefore, we suspect that a model with increased cardiac pericyte loss would have an even more pronounced phenotype. Given that patients experience different viral loads, it would be important to investigate different viral loads and the effect on the development of HFpEF after recovery.

Our results highlight that the cardiac pericyte population can recover in the absence of metabolic comorbidities. Pericytes can migrate and make extensions to replace lost pericytes or recover by proliferation ${ }^{28,32}$. Which of these mechanisms is occurring after SARS-CoV-2 infection is not clear. We can, however, show that pericyte recovery is inhibited by metabolic comorbidities. As such, our results indicate that controlling co-morbidities in COVID19 patients is of primary importance. 
Cardiac fibrosis was present in our HFpEF hamster model, as well as in the infected hamsters, regardless of co-treatment with HFD/L-NAME. Cardiac fibrosis has been demonstrated by cardiovascular magnetic resonance techniques after SARS-CoV-2 infection, even months later in young patients with mild or no symptoms during infection ${ }^{33}$. Similarly, a third of COVID19 patients show increased late gadolinium enhancement (LGE) ${ }^{6,7}$. LGE increase can indicate myocyte necrosis, myocardial oedema, myocardial scar tissue, and focal areas of fibrosis ${ }^{34}$. At the same time, increased LGE predicts mortality in viral myocarditis ${ }^{34}$. Our results suggest that the increased LGE observed after SARS-CoV-2 infection is likely due to increased cardiac fibrosis.

Cardiac pericytes were lost, but they may also be hypersensitive to pro-relaxation signals. Pericytes provide a baseline tone to capillaries and loss of pericytes induces capillary dilatation ${ }^{32}$. Localized loss of pericyte tone increases blood flow to a region ${ }^{35}$, however, massive loss of microvascular tone induces reductions in blood flow ${ }^{36}$. Interestingly, loss of vessel perfusion has been noted upon SARS-CoV-2 infection ${ }^{37}$. Circulating levels of the pericyte vasodilator adenosine increase rapidly during any viral infection ${ }^{38,39}$. Thus, the combination of reduced pericyte numbers and a hypersensitivity to pro-relaxation stimuli may not be beneficial in this case. The receptors for adenosine are also expressed on immune cells, where their activation reduces the intensity of the immune response ${ }^{40}$. If immune cells also upregulated $A D O R A 1 / 2$ in response to SARS-CoV-2 spike protein exposure, then adenosine would lead to a more moderate immune response, something of benefit in the response to SARS-CoV-2 infection. Overall, it is unclear whether ADORA1/2 expression changes represent an overall benefit or detriment. A common variant in adenosine monophosphate deaminase (rs17602729, AMPD1) leads to increased circulating 
adenosine levels ${ }^{41}$ and is present in $20 \%$ of the population ${ }^{42}$. The possible significance of these in vitro results could be confirmed by studying this population and whether they are protected or more sensitive to SARS-CoV-2 infection.

Overall, our results show that SARS-CoV-2 infection primarily targets pericytes in the heart. Microvascular dysfunction and fibrosis are hallmarks of HFpEF. We therefore emphasize the need to manage metabolic comorbidities in COVID19 patients.

\section{MATERIALS}

\section{Animal handling}

Experiments were performed according to the European Directive (2010/63/EU) and approved by the Animal Care and Use Committee of KU Leuven (P217/2017 and P083/2020). Female Syrian hamsters were inoculated intranasally with $2 \times 10^{6}$ TCID50 SARSCoV-2, strain BetaCov/Belgium/GHB-03021/2020 (EPI ISL 407976|2020-02-03), recovered from a nasopharyngeal swab from a patient who returned from Wuhan in February 2020, and sacrificed after $4 \mathrm{dpi}$ or $14 \mathrm{dpi}{ }^{15,16}$. Hamsters received either a regular chow diet or a combination of HFD ( $60 \%$ fat, $20 \%$ proteins, $20 \%$ carbohydrates; D12492, Research Diet Inc.) with L-NAME (0.5 g/L; N5751, Sigma Aldrich) dissolved in the drinking water, as indicated.

\section{Transthoracic echocardiography}

Hamsters were anaesthetized with an intraperitoneal injection of $100 \mathrm{mg} / \mathrm{kg}$ ketamine (Nimatek, Eurovet) and $10 \mathrm{mg} / \mathrm{kg}$ xylazine (Xyl-M, V.M.D. nv/sa) ${ }^{43}$. Complete transthoracic 2D M-mode echocardiography was performed using a MS 250 transducer (13-24 MHz) 
connected to a Vevo 2100 echocardiograph (Visual Sonics). Hamsters were placed in a supine position on a heating pad to maintain core body temperature between $37.5-37.7^{\circ} \mathrm{C}$, measured using a rectal probe. Electrocardiography recordings were performed to monitor heart and breathing rate. If needed, anesthesia dosage was modified to ensure constant heart rates between all hamsters. HR and cardiac dimensions were assessed on the parasternal short-axis M-mode imaging. $\mathrm{CO}, \mathrm{EF}, \mathrm{LV}$ mass, and FS were calculated based on parasternal short-axis M-mode recordings. Left ventricular filling was assessed by pulsed wave Doppler trans-mitral flow velocity tracings, including peak E and A wave velocities, MV DT, and IVRT. Myocardial E' and A' diastolic peak velocity was measured by tissue Doppler imaging at the lateral mitral annulus. $E / A, E / A^{\prime}, E / E^{\prime}$, and $E^{\prime} / A^{\prime}$ ratios were calculated. At least three stable cardiac cycles were averaged for all measurements. More details are available in the online Supplemental Materials.

\section{Organ collection}

Hamsters were sacrificed at $4 \mathrm{dpi}$, or after echocardiography at $14 \mathrm{dpi}$. Hamsters were euthanized by excision of the heart. Approximately 5-30 mg of the apex of the heart was placed in RTK buffer for RNA extraction, the remaining was fixed overnight in $4 \%$ paraformaldehyde (PFA).

\section{SARS-CoV-2 RT-qPCR and end-point titrations}

Hamsters tissues were homogenized with bead disruption (Precellys) in $350 \mu \mathrm{L}$ RLT buffer (RNeasy Mini kit, 74004 Qiagen) and RNA was extracted according to the manufacturer's instructions. RT-qPCR was performed on a LightCycler96 platform (Roche) with iTaq Universal Probes One-Step RT-qPCR kit (1725140, BioRad) with N2 primers and probes 
targeting the nucleocapsid ${ }^{16}$. Standards of SARS-CoV-2 cDNA (IDT) were used to quantitatively express viral load as genome copies per mg tissue. For end-point titrations, Vero E6 cells (African green monkey kidney, ATCC CRL-1586) were cultured in minimal essential medium (15188319, Gibco) supplemented with 10\% fetal bovine serum, 1\% Lglutamine (25030149, Gibco) and 1\% bicarbonate (25080094, Gibco). Homogenized lung tissue in minimal essential medium was titrated on confluent Vero E6 cells in 96-well plates. Viral titers were calculated with the Reed and Muench method and expressed as $50 \%$ tissue culture infectious dose $\left(\mathrm{TCID}_{50}\right)$ per mg tissue.

\section{In situ hybridization}

In situ hybridization was performed as described previously ${ }^{44}$ using SARS-CoV-2 anti-sense specific probes (3' ATTAACCCTCACTAAAGGGATTTGGTGGACCCTCAGATTC 5') and SARS-CoV2 sense specific probes ( $3^{\prime}$ TAATACGACTCACTATAGGGGCGCGACATTCCGAAGAA 5') targeting the positive-sense and negative-sense genomic viral RNA, respectively. In brief, after slides were deparaffinized in xylene and rehydrated through a series of graded ethanol, epitope retrieval was performed for $5 \mathrm{~min}$ in Proteinase $\mathrm{K}\left(3115828001\right.$, Sigma) at $37^{\circ} \mathrm{C}$, followed by post-fixation in 4\% PFA for 20 minutes. Slides were incubated with the antisense and sense probes overnight at $70^{\circ} \mathrm{C}$, with a final concentration of $1 \mu \mathrm{g} / \mathrm{ml}$. Slides were washed through a series of washing buffers and treated with $100 \mu \mathrm{g} / \mathrm{mL}$ RNAse A (EN0531, Thermofisher) for 30 min. Slides were blocked with 2\% Blocking Reagent (11096176001, Roche) for 1 hour and incubated overnight with anti-digoxigenin-AP Fab fragments antibody (1:2000, 11093274910, Roche) at $4^{\circ} \mathrm{C}$. Slides were washed through a series of washing buffers and staining was visualized by BM purple AP substrate (11442074001, Roche) at room temperature, until staining was sufficient. 


\section{Patient characterization}

After routine non-invasive diagnostic work-up and angiography had failed to elucidate any specific cause of heart failure like-symptoms, all patients underwent endomyocardial biopsy $(E M B)$ and left heart catheterization in a standardized manner, as previously described ${ }^{45}$. All patients provided written consent for the procedures (local ethic vote number: EA2/140/16 and EA2/066/20). The investigation conforms with the principles outlined in the Declaration of Helsinki ${ }^{46}$.

Three different groups of patients were investigated and compared:

1. Patients served as controls where a cardiac cause for their chest discomfort were

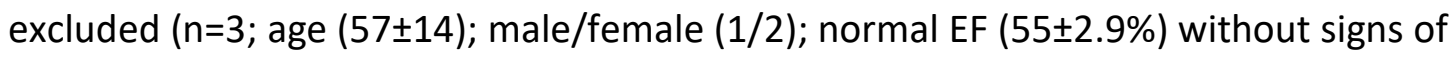
LV diastolic dysfunction and regular NT-pro BNP levels.

2. Symptomatic patients with classical signs of HFpEF, LV diastolic dysfunction, and abnormal NT-proBNP levels, served as a group where a viral presence and / or a significant cardiac inflammation were excluded ( $n=3$; age (54 \pm 7.0$)$; male/female (2/1); preserved EF (55 $\pm 3.2 \%))$.

3. Symptomatic patients, who recovered form COVID-19 ( $n=3 ; 5 \pm 2$ weeks post-COVID-

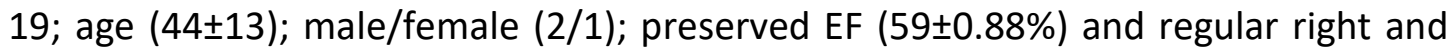
left ventricular wall motions, absence of SARS-CoV2 genome in EMB). In detail, they suffered from exercise intolerance (NYHA II-III), and atypical angina but showed regular NT-pro BNP levels. Spiral CTs excluded lung embolization. Cardiac MRI analyses were positive for post-inflammatory abnormalities (positive T2 mapping), pericardial inflammation or microvascular dysfunction, respectively. None of these patients had an increase in extracellular volume, indicating absence of cardiac fibrosis. 
Presence of other viruses was excluded, whereas subacute lymphocytic myocarditis was diagnosed in 1 out of the 3 patients.

\section{Histological analysis}

All antibodies and concentrations are listed in Supplemental Table S2. EMB were fixed in $10 \%$ neutral buffered formalin overnight at $4^{\circ} \mathrm{C}$ (classical protocol) or at room temperature to avoid potential SARS-CoV2 infection. Four $\mu \mathrm{m}$ sections were stained with anti-CD34 antibodies, $\alpha$ SMA-Cy3 antibody, and NG2 antibody. Epitope retrieval was performed for CD34 and NG2 stainings by using citrate buffer (0.1M Target Retrieval Solution; DAKO S1699, Agilent). Amplification of CD34 was performed using a Tyramide Superboost kit (Alexa Fluor 488 Tyramide SuperBoostTM Kit, B40922, Invitrogen).

For hamster cardiac tissue, four $\mu \mathrm{m}$ paraffin sections were cut and deparaffinized. For laminin staining, proteinase K epitope retrieval was used (1/500 in PBS, 3115828001, Sigma Aldrich). For NG2, PDGFR- $\beta$, and $\alpha$ SMA staining citrate buffer (0.1M Target Retrieval Solution, DAKO S1699, Agilent) was used.

\section{In vitro stimulation of pericytes}

Human immortalized pericytes (CL 05008-CLTH, Celther) were cultured in DMEM high glucose medium (41965039, Thermofisher) supplemented with penicillin/streptomycin. Cells were exposed to $50 \mathrm{ng} / \mathrm{ml}$ Recombinant SARS-CoV-2 S Protein RBD Fc Chimera, CF (10499-CV, R\&D Systems) for $24 \mathrm{~h}$ to induce ACE2 internalization. Three samples per condition were stained with Annexin V (1/9; A13202, Thermofisher) to assess the number of apoptotic cells. Sequences of qPCR primers are given in Supplemental Table S3. 


\section{Statistical analysis}

Results are presented as mean \pm SEM. Statistical analysis was performed using GraphPad 8 Prism. Variance was tested with F-test $(p<0.05)$ and normality was tested with Shapiro-Wilk test $(p<0.05)$. Biological outliers were picked up based on Grubb's test $(p<0.05)$. In vivo data were analyzed using a one-way ANOVA with Dunnett's post hoc test $(p<0.05 *, 0.01$ $* *, 0.001 * * *)$.

\section{ACKNOWLEDGEMENTS}

EAVJ was supported by the Fonds Wetenschappelijk Onderzoek [G091018N, G0B5920N, $1160718 \mathrm{~N}, 1107721 \mathrm{~N}$ ], by internal funding from the KU Leuven [IDN/19/031, C14/19/095]. This project has also received funding from the European Union's Horizon 2020 research and innovation program to EAVJ under grant agreement No 848109. JN was supported by funding from the Covid-19-Fund KU Leuven / University Hospitals Leuven and the COVID-19 call of the Research Foundation Flanders (FWO) (grant G0G4820N), the European Union's Horizon 2020 research and innovation program under Grant Agreement 101003627 (Swift COronavirus therapeutics REsponse project) and funding from the Bill and Melinda Gates Foundation under Grant Agreement INV-00636.

\section{AUTHOR CONTRIBUTION}

MD performed experiments, designed experiments, analysed data and wrote the manuscript. LL performed experiments, designed experiments, analysed data and edited the 
manuscript. IC analysed data and edited the manuscript. RB performed experiments and edited the manuscript. JR analysed data and edited the manuscript. SJS performed experiments and edited the manuscript. NG performed experiments. ML performed experiments. PV designed experiments and edited the manuscript. SVL designed experiments, and edited the manuscript. SH designed experiments and edited the manuscript. CT performed experiments, designed experiments, and edited the manuscript. EAVJ designed experiments, analysed data and wrote the manuscript.

\section{DATA AVAILABILITY STATEMENT}

All data are available upon request.

\section{COMPETING INTERESTS}

The authors declare no competing or financial interests. 


\section{REFERENCES}

1. Basso, C., et al. Pathological features of COVID-19-associated myocardial injury: a multicentre cardiovascular pathology study. Eur Heart J (2020).

2. Shi, S., et al. Association of Cardiac Injury With Mortality in Hospitalized Patients With COVID-19 in Wuhan, China. JAMA Cardiol 5, 802-810 (2020).

3. Shi, S., et al. Characteristics and clinical significance of myocardial injury in patients with severe coronavirus disease 2019. Eur Heart J 41, 2070-2079 (2020).

4. Giustino, G., et al. Characterization of Myocardial Injury in Patients With COVID-19. J Am Coll Cardiol 76, 2043-2055 (2020).

5. Toraih, E.A., et al. Association of cardiac biomarkers and comorbidities with increased mortality, severity, and cardiac injury in COVID-19 patients: A metaregression and decision tree analysis. J Med Virol (2020).

6. Huang, L., et al. Cardiac Involvement in Patients Recovered From COVID-2019 Identified Using Magnetic Resonance Imaging. JACC Cardiovasc Imaging (2020).

7. Puntmann, V.O., et al. Outcomes of Cardiovascular Magnetic Resonance Imaging in Patients Recently Recovered From Coronavirus Disease 2019 (COVID-19). JAMA Cardiol (2020).

8. Bavishi, C., et al. Acute myocardial injury in patients hospitalized with COVID-19 infection: A review. Prog Cardiovasc Dis (2020).

9. Puelles, V.G., et al. Multiorgan and Renal Tropism of SARS-CoV-2. N Engl J Med 383, 590-592 (2020).

10. Tostanoski, L.H., et al. Ad26 vaccine protects against SARS-CoV-2 severe clinical disease in hamsters. Nat Med (2020).

11. Chen, L., Li, X., Chen, M., Feng, Y. \& Xiong, C. The ACE2 expression in human heart indicates new potential mechanism of heart injury among patients infected with SARS-CoV-2. Cardiovasc Res 116, 1097-1100 (2020).

12. He, L., et al. Pericyte-specific vascular expression of SARS-CoV-2 receptor ACE2 implications for microvascular inflammation and hypercoagulopathy in COVID-19. bioRxiv doi: 10.1101/2020.05.11.088500(2020).

13. Szekely, Y., et al. Spectrum of Cardiac Manifestations in COVID-19: A Systematic Echocardiographic Study. Circulation 142, 342-353 (2020).

14. Freaney, P.M., Shah, S.J. \& Khan, S.S. COVID-19 and Heart Failure With Preserved Ejection Fraction. JAMA (2020).

15. Kaptein, S.J.F., et al. Favipiravir at high doses has potent antiviral activity in SARSCoV-2-infected hamsters, whereas hydroxychloroquine lacks activity. Proc Natl Acad Sci U S A (2020).

16. Boudewijns, R., et al. STAT2 signaling as double-edged sword restricting viral dissemination but driving severe pneumonia in SARS-CoV-2 infected hamsters. bioRxiv doi: 10.1101/2020.04.23.056838(2020).

17. Kuba, K., et al. A crucial role of angiotensin converting enzyme 2 (ACE2) in SARS coronavirus-induced lung injury. Nat Med 11, 875-879 (2005).

18. Matsugi, T., Chen, Q. \& Anderson, D.R. Adenosine-induced relaxation of cultured bovine retinal pericytes. Invest Ophthalmol Vis Sci 38, 2695-2701 (1997).

19. Hall, C.N., et al. Capillary pericytes regulate cerebral blood flow in health and disease. Nature 508, 55-60 (2014). 
20. Oishi, K., Kamiyashiki, T. \& Ito, Y. Isometric contraction of microvascular pericytes from mouse brain parenchyma. Microvasc Res 73, 20-28 (2007).

21. Tilton, R.G., et al. Pericyte degeneration and acellular capillaries are increased in the feet of human diabetic patients. Diabetologia 28, 895-900 (1985).

22. Corliss, B.A., et al. Pericyte Bridges in Homeostasis and Hyperglycemia. Diabetes 69, 1503-1517 (2020).

23. McMurray, J.J., et al. Heart failure with preserved ejection fraction: clinical characteristics of 4133 patients enrolled in the I-PRESERVE trial. Eur J Heart Fail 10, 149-156 (2008).

24. Zhou, F., et al. Clinical course and risk factors for mortality of adult inpatients with COVID-19 in Wuhan, China: a retrospective cohort study. Lancet 395, 1054-1062 (2020).

25. Schiattarella, G.G., et al. Nitrosative stress drives heart failure with preserved ejection fraction. Nature 568, 351-356 (2019).

26. Nguyen, I.T.N., et al. Both male and female obese ZSF1 rats develop cardiac dysfunction in obesity-induced heart failure with preserved ejection fraction. PLoS One 15, e0232399 (2020).

27. Deckx, S., et al. Extracellular SPARC increases cardiomyocyte contraction during health and disease. PLoS One 14, e0209534 (2019).

28. Chen, W.C., et al. Human myocardial pericytes: multipotent mesodermal precursors exhibiting cardiac specificity. Stem Cells 33, 557-573 (2015).

29. Xu, D., et al. Single-cell Transcriptome Analysis Indicates New Potential Regulation Mechanism of ACE2 and NPs signaling among heart failure patients infected with SARS-CoV-2. medRxiv (2020).

30. Van Linthout, S., Klingel, K. \& Tschope, C. SARS-CoV-2-related myocarditis-like syndromes Shakespeare's question: what's in a name? Eur J Heart Fail 22, 922-925 (2020).

31. Knight, D.S., et al. COVID-19: Myocardial Injury in Survivors. Circulation 142, 11201122 (2020).

32. Berthiaume, A.A., et al. Dynamic Remodeling of Pericytes In Vivo Maintains Capillary Coverage in the Adult Mouse Brain. Cell Rep 22, 8-16 (2018).

33. Clark, D.E., et al. COVID-19 Myocardial Pathology Evaluated Through scrEening Cardiac Magnetic Resonance (COMPETE CMR). medRxiv (2020).

34. Grani, C., et al. Prognostic Value of Cardiac Magnetic Resonance Tissue Characterization in Risk Stratifying Patients With Suspected Myocarditis. J Am Coll Cardiol 70, 1964-1976 (2017).

35. Hartmann, D.A., et al. Brain capillary pericytes exert a substantial but slow influence on blood flow. bioRxiv doi: 10.1101/2020.03.26.008763(2020).

36. Nikolakopoulou, A.M., et al. Pericyte loss leads to circulatory failure and pleiotrophin depletion causing neuron loss. Nat Neurosci 22, 1089-1098 (2019).

37. Damiani, E., et al. Microvascular alterations in patients with SARS-COV-2 severe pneumonia. Ann Intensive Care 10, 60 (2020).

38. Jabs, C.M., Sigurdsson, G.H. \& Neglen, P. Plasma levels of high-energy compounds compared with severity of illness in critically ill patients in the intensive care unit. Surgery 124, 65-72 (1998). 
39. Martin, C., Leone, M., Viviand, X., Ayem, M.L. \& Guieu, R. High adenosine plasma concentration as a prognostic index for outcome in patients with septic shock. Crit Care Med 28, 3198-3202 (2000).

40. Drygiannakis, I., Ernst, P.B., Lowe, D. \& Glomski, I.J. Immunological alterations mediated by adenosine during host-microbial interactions. Immunol Res 50, 69-77 (2011).

41. Riksen, N.P., et al. Augmented hyperaemia and reduced tissue injury in response to ischaemia in subjects with the $34 \mathrm{C}>$ T variant of the AMPD1 gene. Eur Heart $\mathrm{J} \mathbf{2 8}$, 1085-1091 (2007).

42. Morisaki, T., et al. Molecular basis of AMP deaminase deficiency in skeletal muscle. Proc Natl Acad Sci U S A 89, 6457-6461 (1992).

43. Tanaka, D.M., et al. Effect of different anesthetic agents on left ventricular systolic function assessed by echocardiography in hamsters. Braz J Med Biol Res 49, e5294 (2016).

44. Wu, J., Feng, J.Q. \& Wang, X. In Situ Hybridization on Mouse Paraffin Sections Using DIG-Labeled RNA Probes. Methods Mol Biol 1922, 163-171 (2019).

45. Tschope, C., Kherad, B. \& Schultheiss, H.P. How to perform an endomyocardial biopsy? Turk Kardiyol Dern Ars 43, 572-575 (2015).

46. Rickham, P.P. Human Experimentation. Code of Ethics of the World Medical Association. Declaration of Helsinki. Br Med J 2, 177 (1964). 


\section{FIGURES}
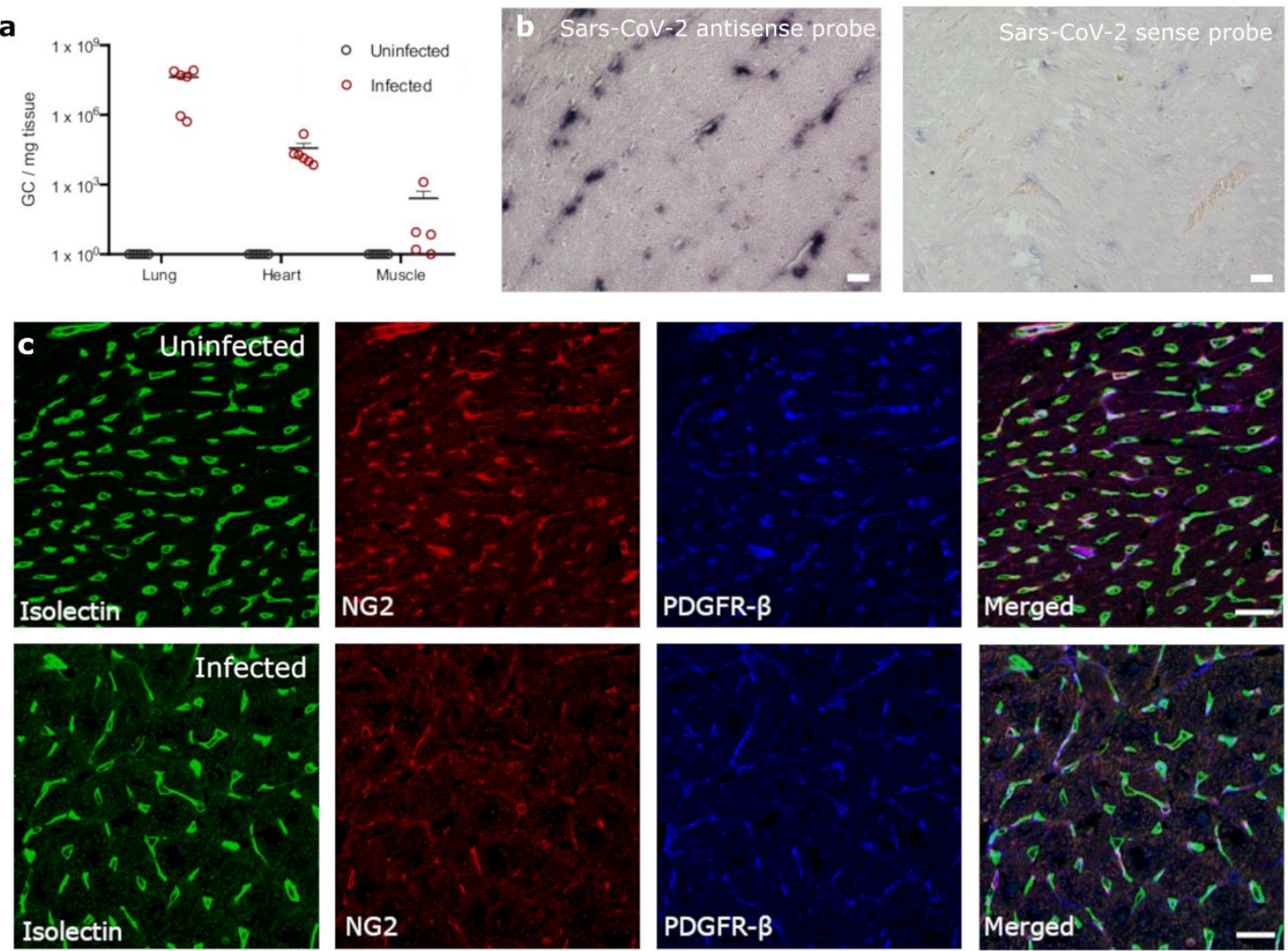

d
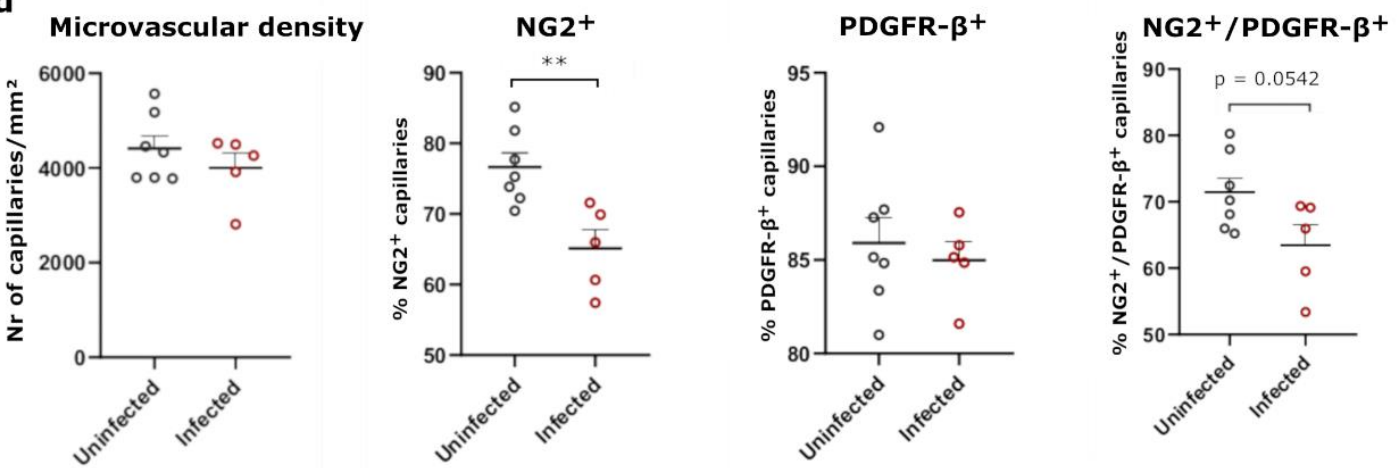

Figure 1. SARS-CoV-2 infected hamsters lose their $\mathrm{NG2}^{+}$pericytes at 4 days post infection.

Hamsters were infected with SARS-CoV-2 virus and sacrificed 4 days post infection, at the peak of viral load. (a) Viral RNA levels were quantified by RT-qPCR in lung, heart, and muscle from infected and uninfected hamsters. (b) SARS-CoV-2 infection was analyzed by in situ hybridization techniques (c) Heart sections of control and infected hamsters were stained for isolectin (green), NG2 (red), and PDGFRß (blue) to analyze percent pericyte positive 
capillaries (d). HFD, high fat diet; L-NAME, $N$ $~$ Nitro L arginine methyl ester; NG2, neuronglial antigen 2; PDGFRß, platelet-derived growth factor receptor beta. Values are presented as mean \pm SEM with $n=7$ (control) and 5 (SARS-CoV-2 infected hamsters). Statistical analysis was performed using a two-tailed students t-test, with $* p<0.05$ and $* * p<0.01$. Scalebar represents $20 \mu \mathrm{m}(\mathbf{b}, \mathbf{c}, \mathbf{d})$. 

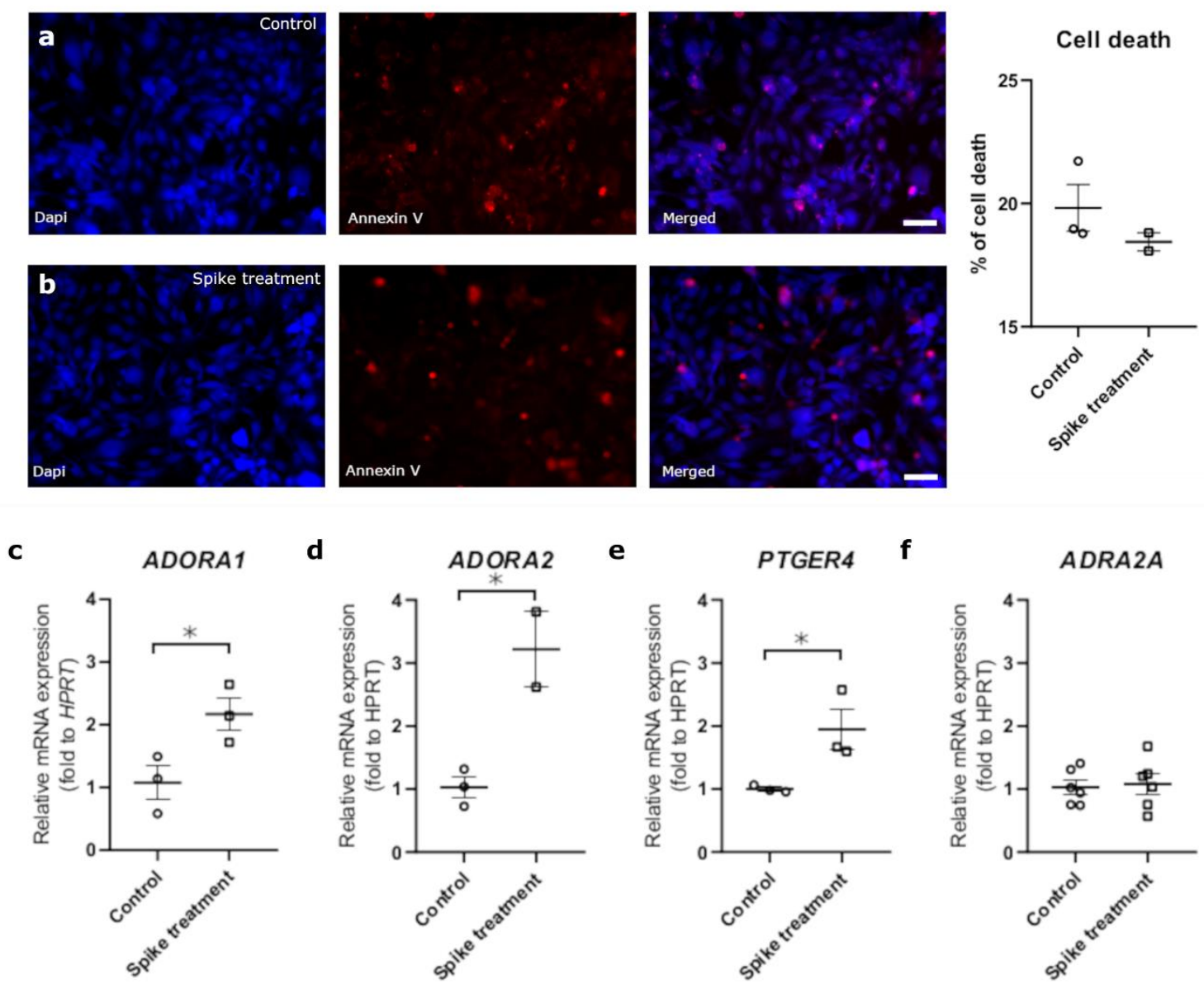

Figure 2. SARS-CoV-2 spike protein exposure makes immortalized pericytes more susceptible for relaxation, without inducing apoptosis. Immortalized pericytes were exposed to $50 \mathrm{ng} / \mathrm{ml}$ SARS-CoV-2 spike protein for $24 \mathrm{~h}$. (a-b) Pericytes were stained for Annexin V (red) and ToPro (blue), with double positive cells indicating apoptosis. (c-f) RNA levels for ADORA1 (c), ADORA2 (d) PTGER4 (e) and ADRA2A (f) were analyzed by RT-qPCR. ADORA1, adenosine A1 receptor; ADORA2, adenosine A2A receptor; PTGER4, prostaglandin E2 receptor 4; ADRA2A, Adrenoceptor Alpha 2A receptor. Values are presented as mean \pm SEM with $n=2-3$. Statistical analysis was performed using a two-tailed students t-test, with $* p<0.05$. Scalebar represents $100 \mu \mathrm{m}(\mathbf{a}-\mathbf{b})$. 

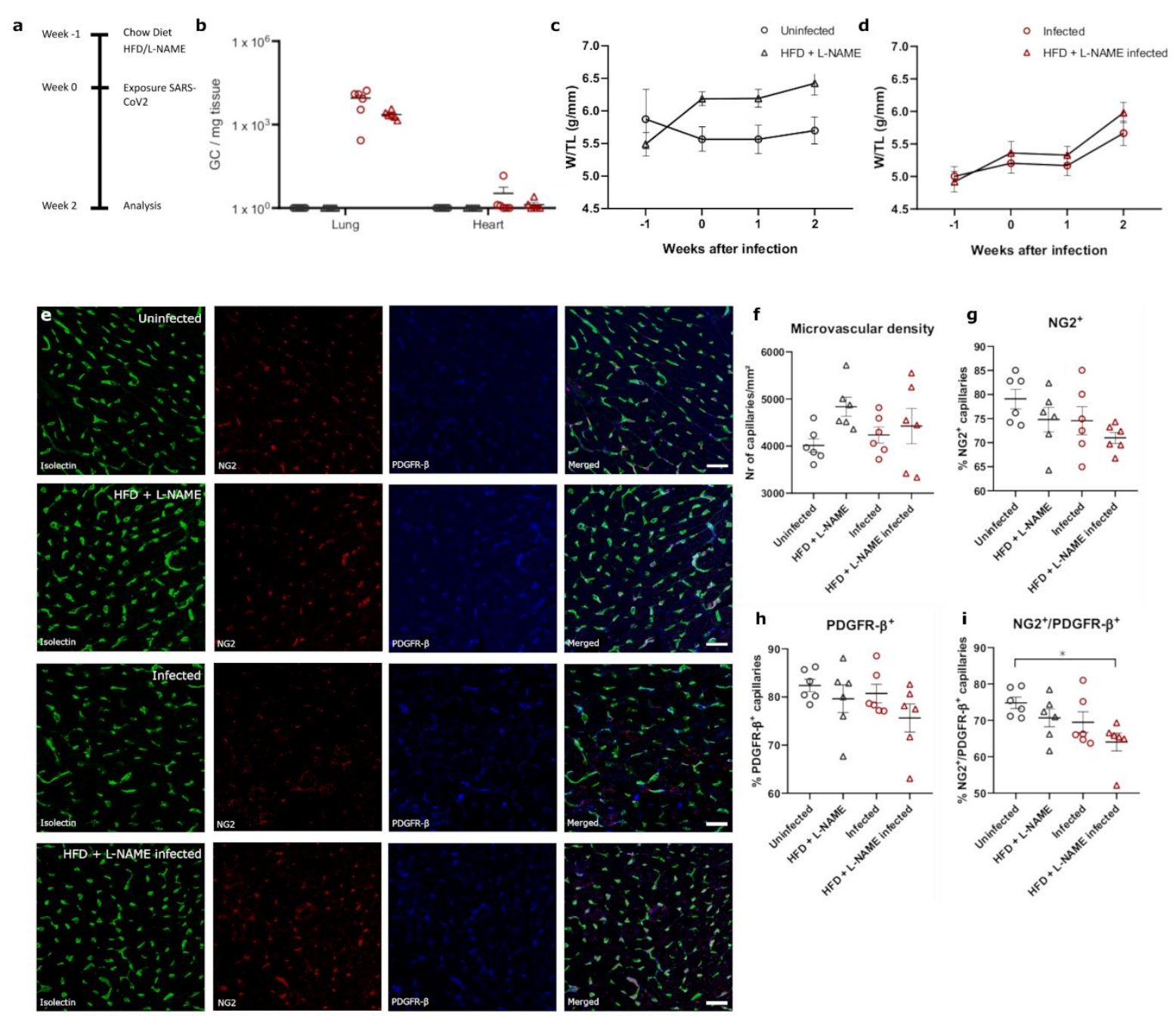

Figure 3. Hamsters on HFD + L-NAME diet do not recover their pericyte coverage after

SARS-CoV-2 infection. (a) Twelve-week-old hamsters received chow diet or HFD + L-NAME diet one week prior to SARS-CoV-2 infection. Hamsters were subjected to a cardiac echography and sacrificed two weeks after SARS-CoV-2 infection. (b) Viral RNA levels were quantified by RT-qPCR in lung and heart of all hamster groups. (c-d) Body weights were followed up over the course of the experiment and normalized to tibia length. (e) Heart sections of control and infected hamsters were stained for isolectin (green), NG2 (red), and PDGFRß (blue) to analyze pericyte coverage (f-i). GC, genome copies; HFD, high fat diet; LNAME, $N$ Y Nitro L arginine methyl ester; PDGFRß, platelet-derived growth factor receptor 
beta; TL, tibia length; $W$, weight. Values are presented as mean \pm SEM with $n=6$. Statistical analysis of body weights was performed using a two-way ANOVA with a Dunnett's multiple comparison post hoc test with $* p<0.05, * * p<0.01$. All other data was analyzed using a one-way ANOVA followed by a Dunnett's multiple comparisons test, with * $p<0.05$, comparing HFD + L-NAME hamsters or infected hamsters with or without HFD + L-NAME diets to control hamsters. Scalebar represents $20 \mu \mathrm{m}(\mathbf{e})$. 

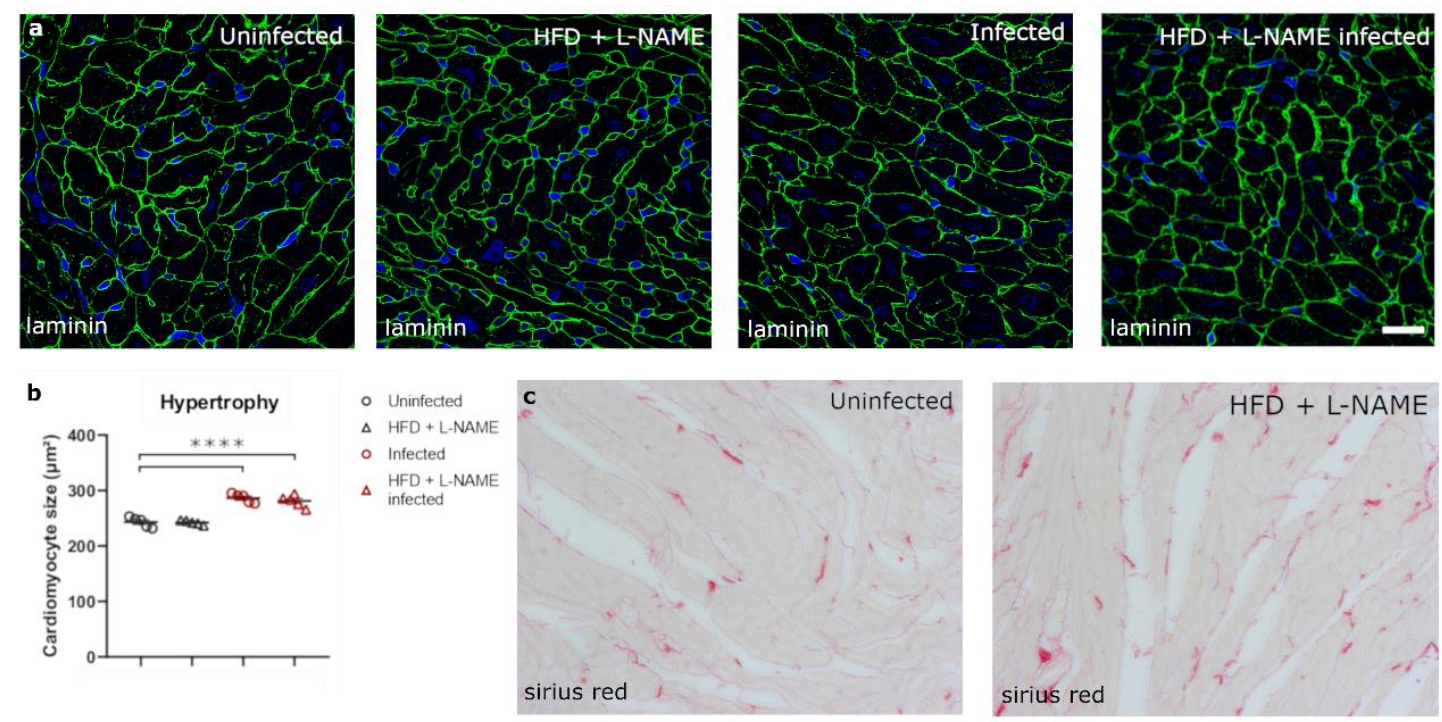

d
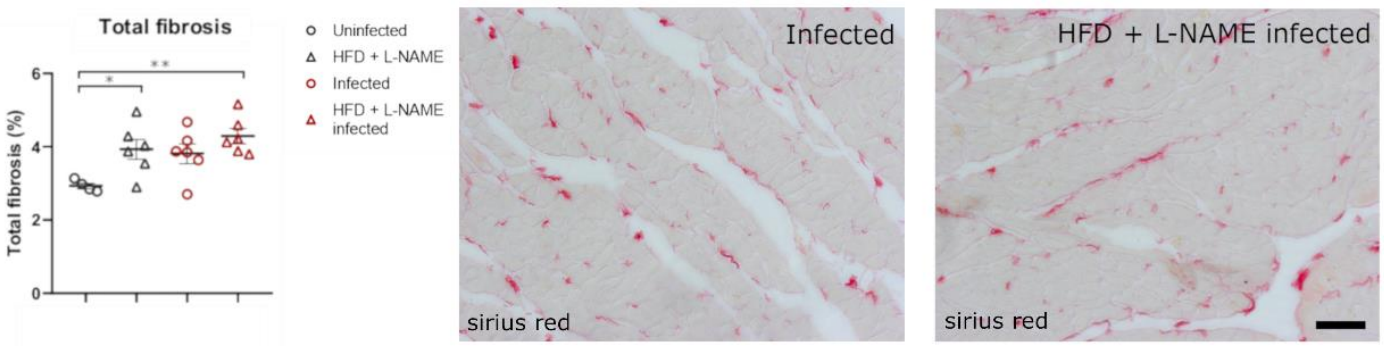

Figure 4. SARS-CoV-2 infected hamsters present with a small cardiomyocyte hypertrophy

and increased cardiac fibrosis. (a) Heart sections of control and infected hamsters, either on regular chow diet or HFD + L-NAME diet, were stained for laminin and Topro3 to quantify cardiomyocyte hypertrophy (b). (c) Heart sections of control and infected hamsters, either on regular chow diet or HFD + L-NAME diet, were stained with Picro Sirius Red to analyze cardiac fibrosis (D). HFD, high fat diet; L-NAME, $N$ $~$ Nitro $L$ arginine methyl ester. Values are presented as mean \pm SEM with $n=6$. Statistical analysis was analyzed using a one-way ANOVA followed by a Dunnett's multiple comparisons test, with * $p<0.05$, comparing HFD + L-NAME hamsters or infected hamsters with or without HFD + L-NAME diets to control hamsters. Scalebar represents $20 \mu \mathrm{m}$ (a), $50 \mu \mathrm{m}$ (c). 

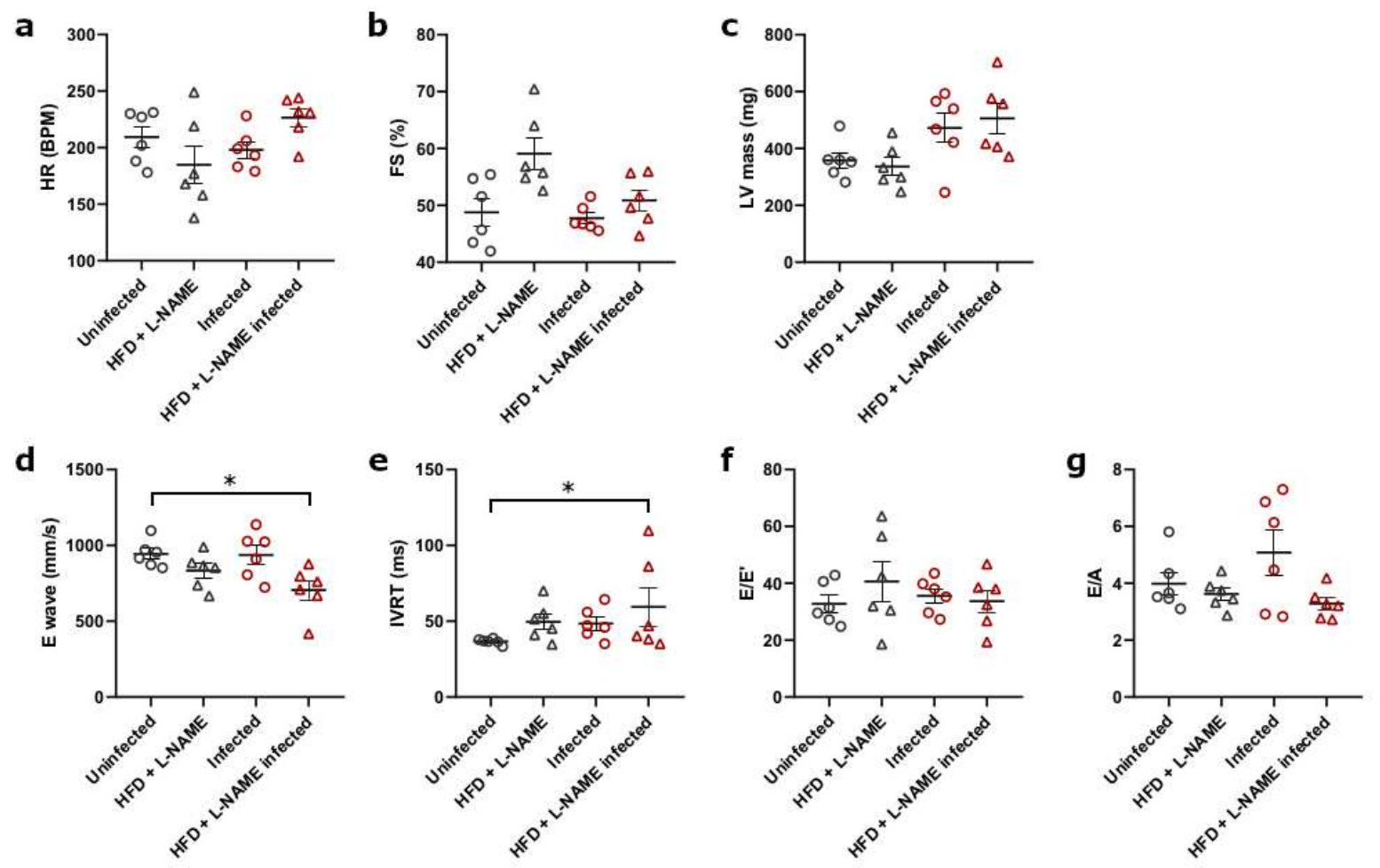

Figure 5. Echocardiography demonstrates an extremely mild diastolic dysfunction in SARS-

CoV-2 infected hamsters on HFD + L-NAME diet. Cardiac function of hamsters was investigated using echocardiography two weeks after SARS-CoV-2 infection. HR (a), FS (b), E wave (c), IVRT (d), E/E' (f), and E/A (g) were analyzed. A, late mitral inflow peak velocity; E, early mitral inflow peak velocity; E', early diastolic mitral annulus peak velocity; FS, fractional shortening; HFD, high fat diet; HR, heart rate; IVRT, isovolumic relaxation time; LNAME, $N \gamma$ Nitro $L$ arginine methyl ester. Values are presented as mean \pm SEM with $n=6$. All data were analyzed using a one-way ANOVA followed by a Dunnett's multiple comparison test with $* p<0.05, * * p<0.01$, comparing HFD + L-NAME hamsters or infected hamsters with or without HFD + L-NAME diets to control hamsters. 

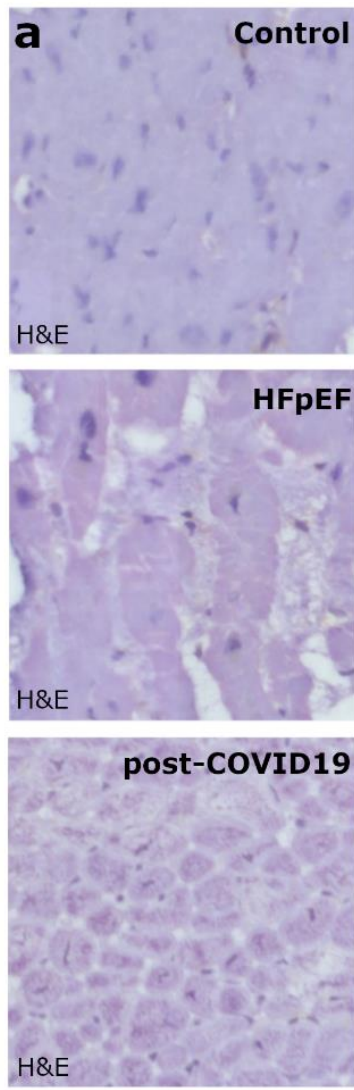
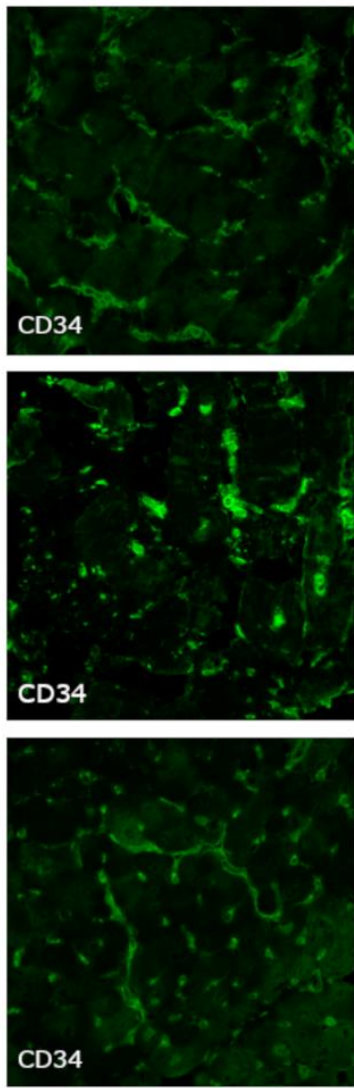

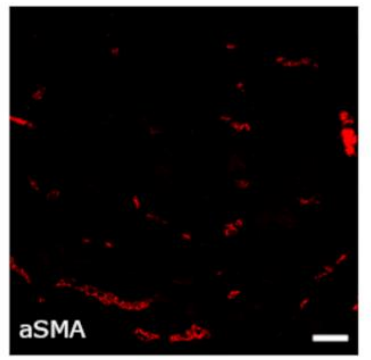

b Microvascular density

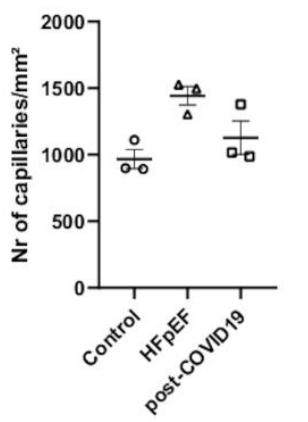

C $\quad$ SSMA ${ }^{\text {low }}$ capillaries

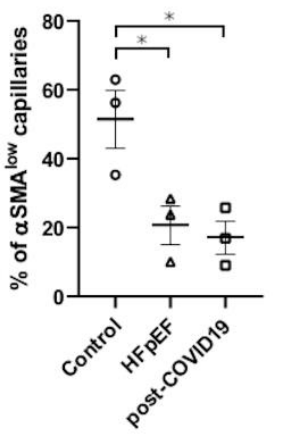

Figure 6. Recovered COVID19 patients lose the same amount of $\alpha S M A^{\text {low }}$ pericytes as patients that develop HFpEF independently of SARS-CoV-2 infection. (a) Heart biopsies from control, HFpEF patients, and recovered COVID19 patients were stained for H\&E, CD34 (green), and $\alpha \mathrm{SMA}$ (red). Microvascular density (b) and the amount of $\alpha \mathrm{SMA}^{\text {low }}$ vessels (c) were analyzed. H\&E, hematoxylin, and eosin; $\alpha \mathrm{SMA}$, alpha smooth muscle actin. Values are presented as mean \pm SEM with $n=3$ per group. Statistical analysis was performed using one-way ANOVA followed by a Dunnett's multiple comparisons test, with * $p<0.05$, comparing HFpEF patients or recovered COVID19 patients to controls. Scalebar represents $20 \mu \mathrm{m}$. 


\section{Figures}

a

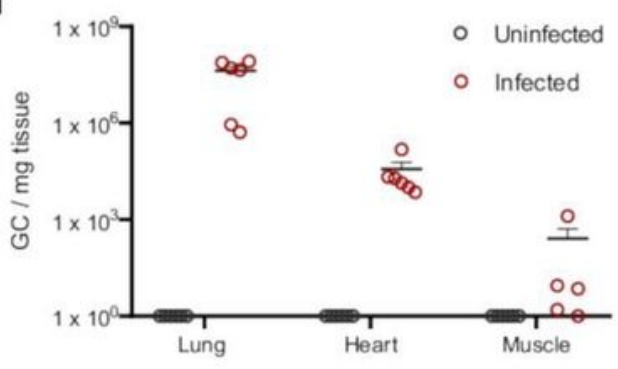

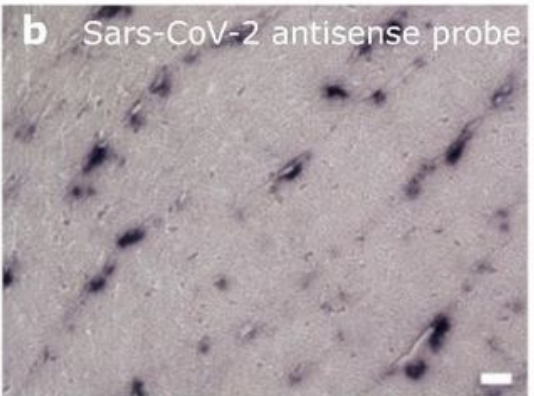
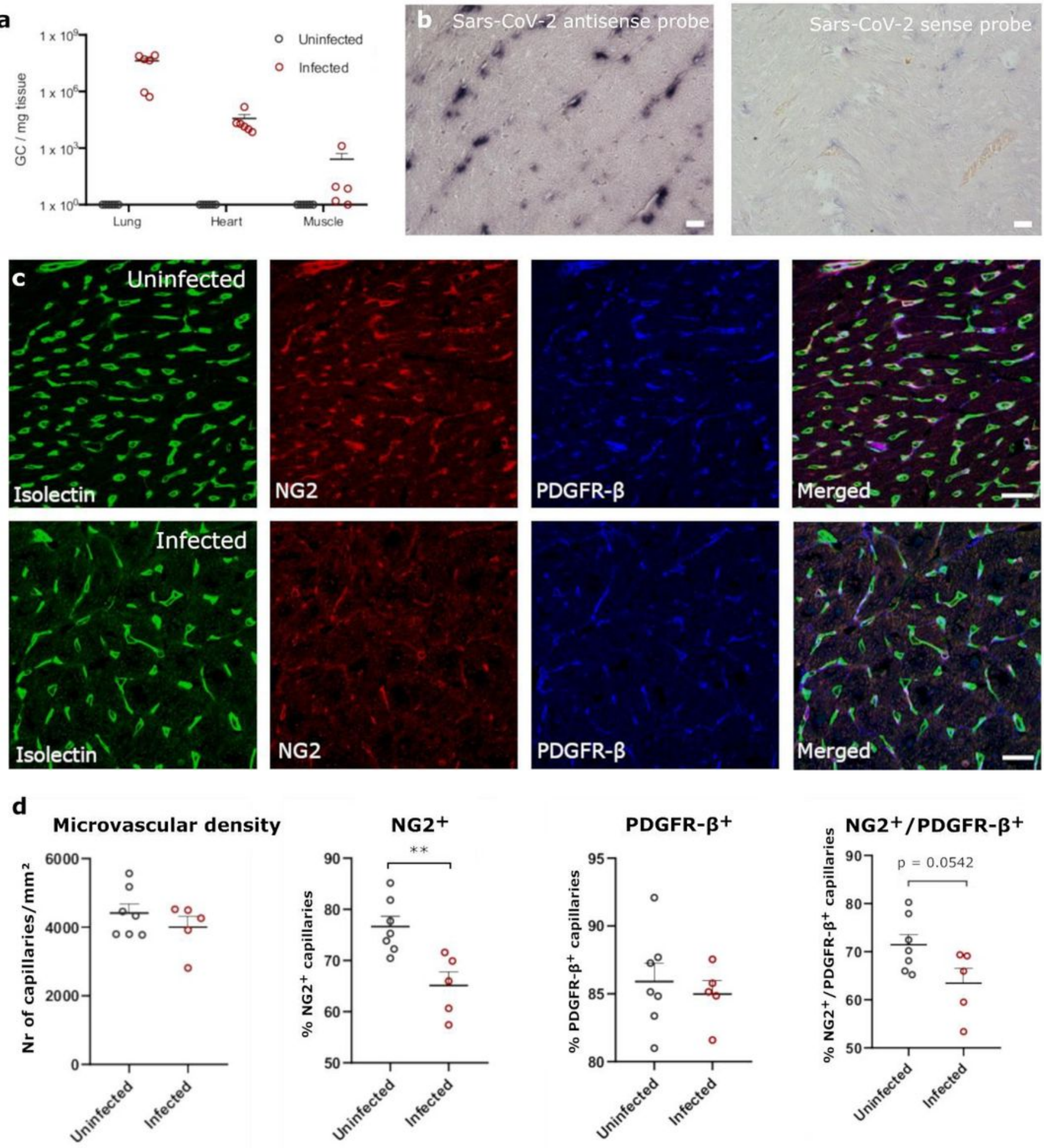

Figure 1

SARS-CoV-2 infected hamsters lose their NG2+ pericytes at 4 days post infection. Hamsters were infected with SARS-CoV-2 virus and sacrificed 4 days post infection, at the peak of viral load. (a) Viral RNA levels were quantified by RT-qPCR in lung, heart, and muscle from infected and uninfected hamsters. (b) SARS- 
CoV-2 infection was analyzed by in situ hybridization techniques (c) Heart sections of control and infected hamsters were stained for isolectin (green), NG2 (red), and PDGFRß (blue) to analyze percent pericyte positive capillaries (d). HFD, high fat diet; L-NAME, N y Nitro L arginine methyl ester; NG2, neuronglial antigen 2; PDGFR $\beta$, platelet-derived growth factor receptor beta. Values are presented as mean \pm SEM with $\mathrm{n}=7$ (control) and 5 (SARS-CoV-2 infected hamsters). Statistical analysis was performed using a two-tailed students t-test, with * $p<0.05$ and $* * p<0.01$. Scalebar represents $20 \mu \mathrm{m}(\mathrm{b}, \mathrm{c}, \mathrm{d})$.
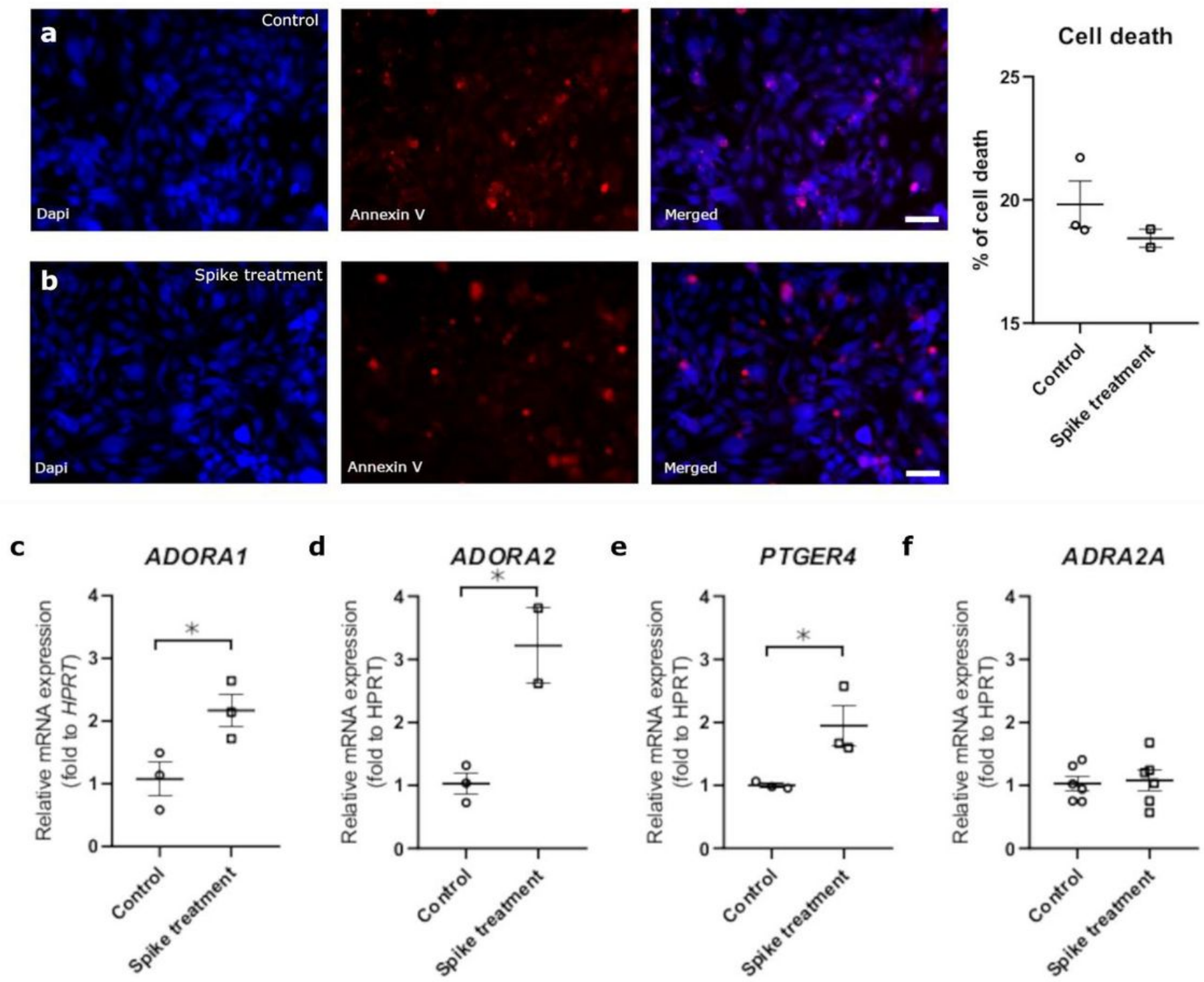

\section{Figure 2}

SARS-CoV-2 spike protein exposure makes immortalized pericytes more susceptible for relaxation, without inducing apoptosis. Immortalized pericytes were exposed to $50 \mathrm{ng} / \mathrm{ml} \mathrm{SARS-CoV-2} \mathrm{spike} \mathrm{protein}$ for 24h. (a-b) Pericytes were stained for Annexin V (red) and ToPro (blue), with double positive cells indicating apoptosis. (c-f) RNA levels for ADORA1 (c), ADORA2 (d) PTGER4 (e) and ADRA2A (f) were analyzed by RT-qPCR. ADORA1, adenosine A1 receptor; ADORA2, adenosine A2A receptor; PTGER4, prostaglandin E2 receptor 4; ADRA2A, Adrenoceptor Alpha 2A receptor. Values are presented as mean \pm 
SEM with $n=2-3$. Statistical analysis was performed using a two-tailed students t-test, with * $p<0.05$. Scalebar represents $100 \mu \mathrm{m}(a-b)$.
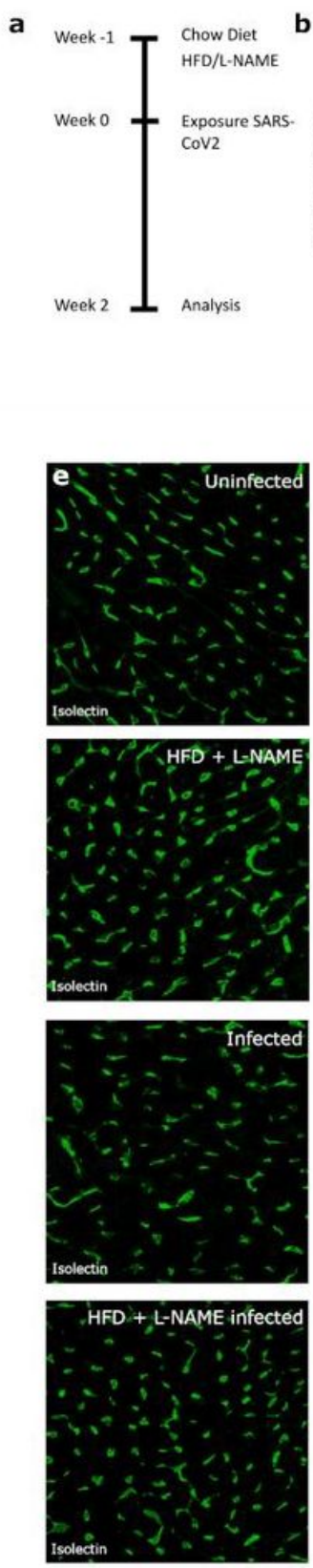
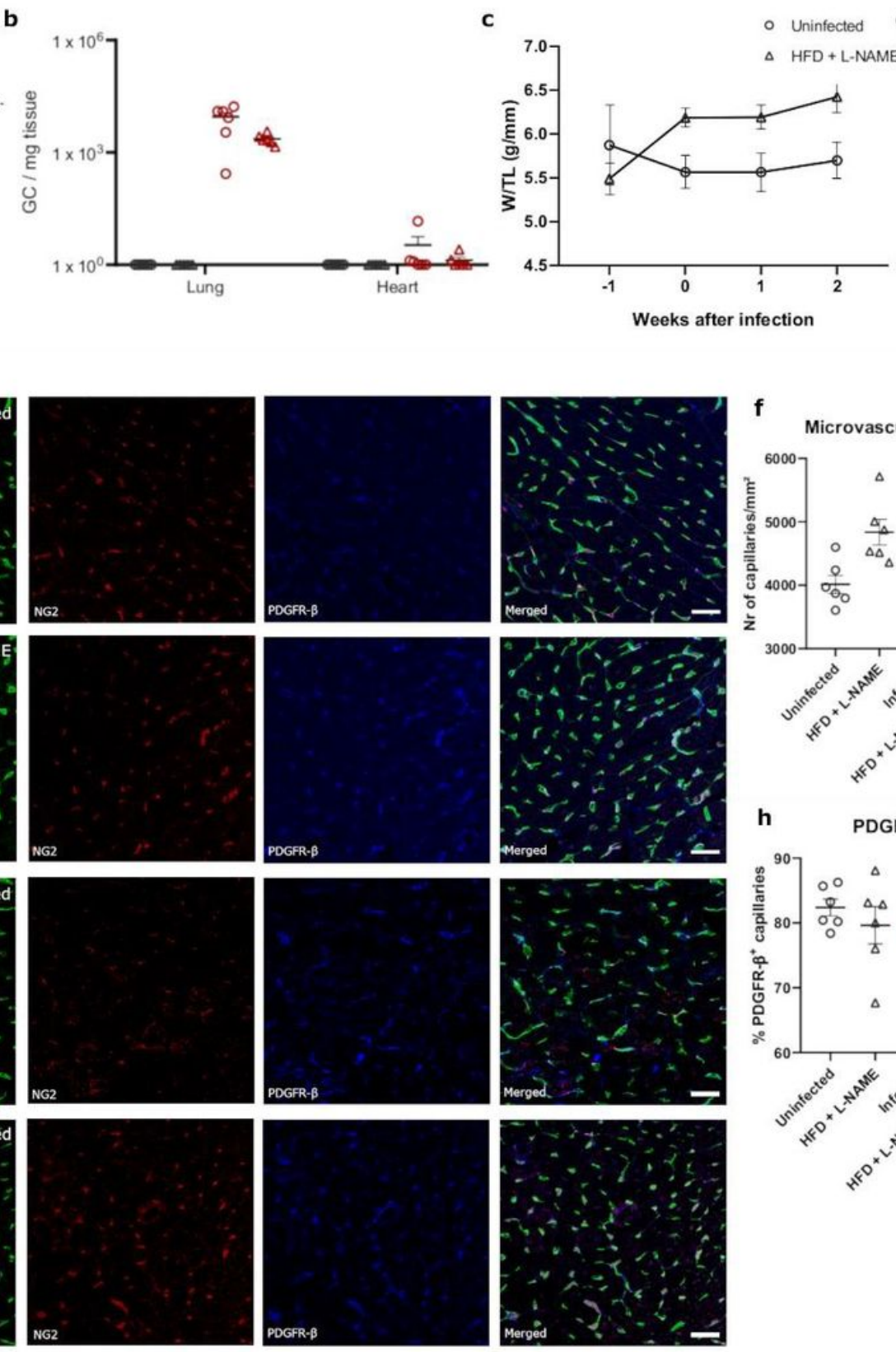
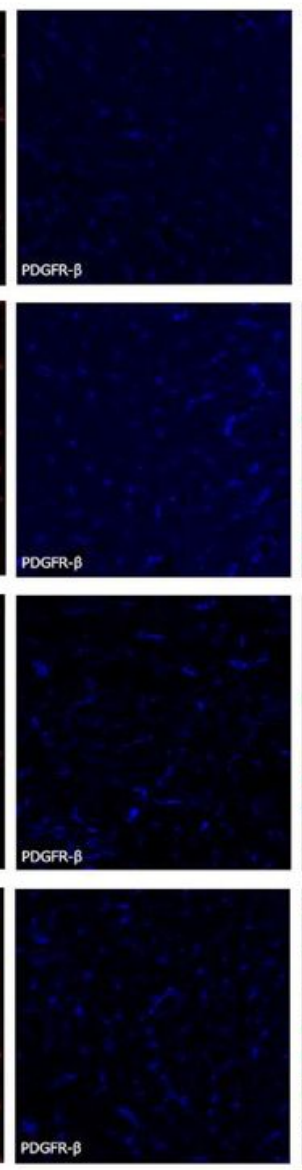
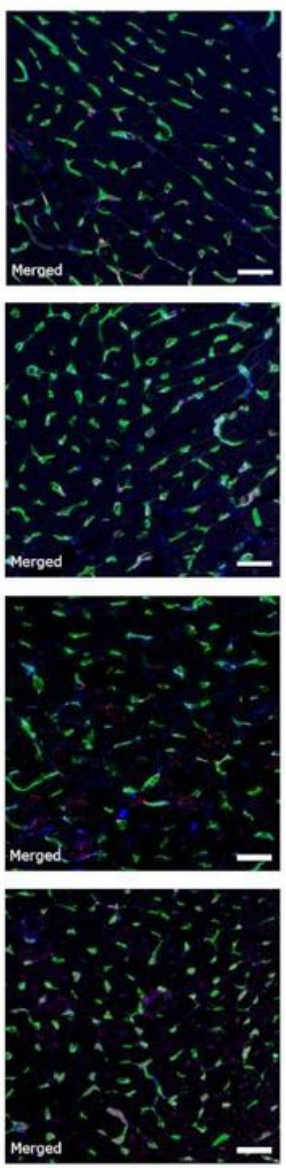
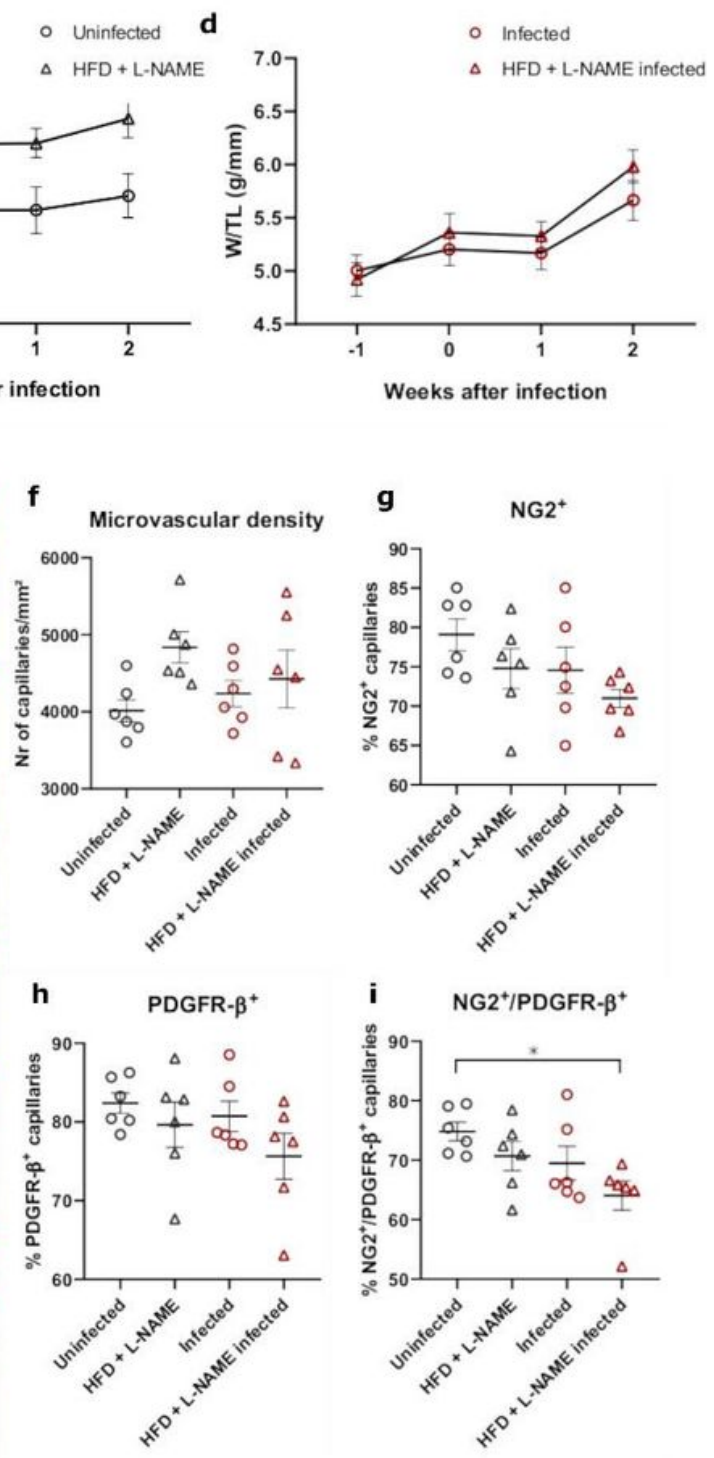

\section{Figure 3}

Hamsters on HFD + L-NAME diet do not recover their pericyte coverage after SARS-CoV-2 infection. (a) Twelve-week-old hamsters received chow diet or HFD + L-NAME diet one week prior to SARS-CoV-2 infection. Hamsters were subjected to a cardiac echography and sacrificed two weeks after SARS-CoV-2 infection. (b) Viral RNA levels were quantified by RT-qPCR in lung and heart of all hamster groups. (c-d) Body weights were followed up over the course of the experiment and normalized to tibia length. (e) Heart sections of control and infected hamsters were stained for isolectin (green), NG2 (red), and PDGFR $\beta$ (blue) to analyze pericyte coverage (f-i). GC, genome copies; HFD, high fat diet; L-NAME, N y Nitro L 
arginine methyl ester; PDGFR , platelet-derived growth factor receptor beta; TL, tibia length; W, weight. Values are presented as mean \pm SEM with $n=6$. Statistical analysis of body weights was performed using a two-way ANOVA with a Dunnett's multiple comparison post hoc test with * $p<0.05, * \star p<0.01$. All other data was analyzed using a one-way ANOVA followed by a Dunnett's multiple comparisons test, with * $p<0.05$, comparing HFD + L-NAME hamsters or infected hamsters with or without HFD + L-NAME diets to control hamsters. Scalebar represents $20 \mu \mathrm{m}(\mathrm{e})$.
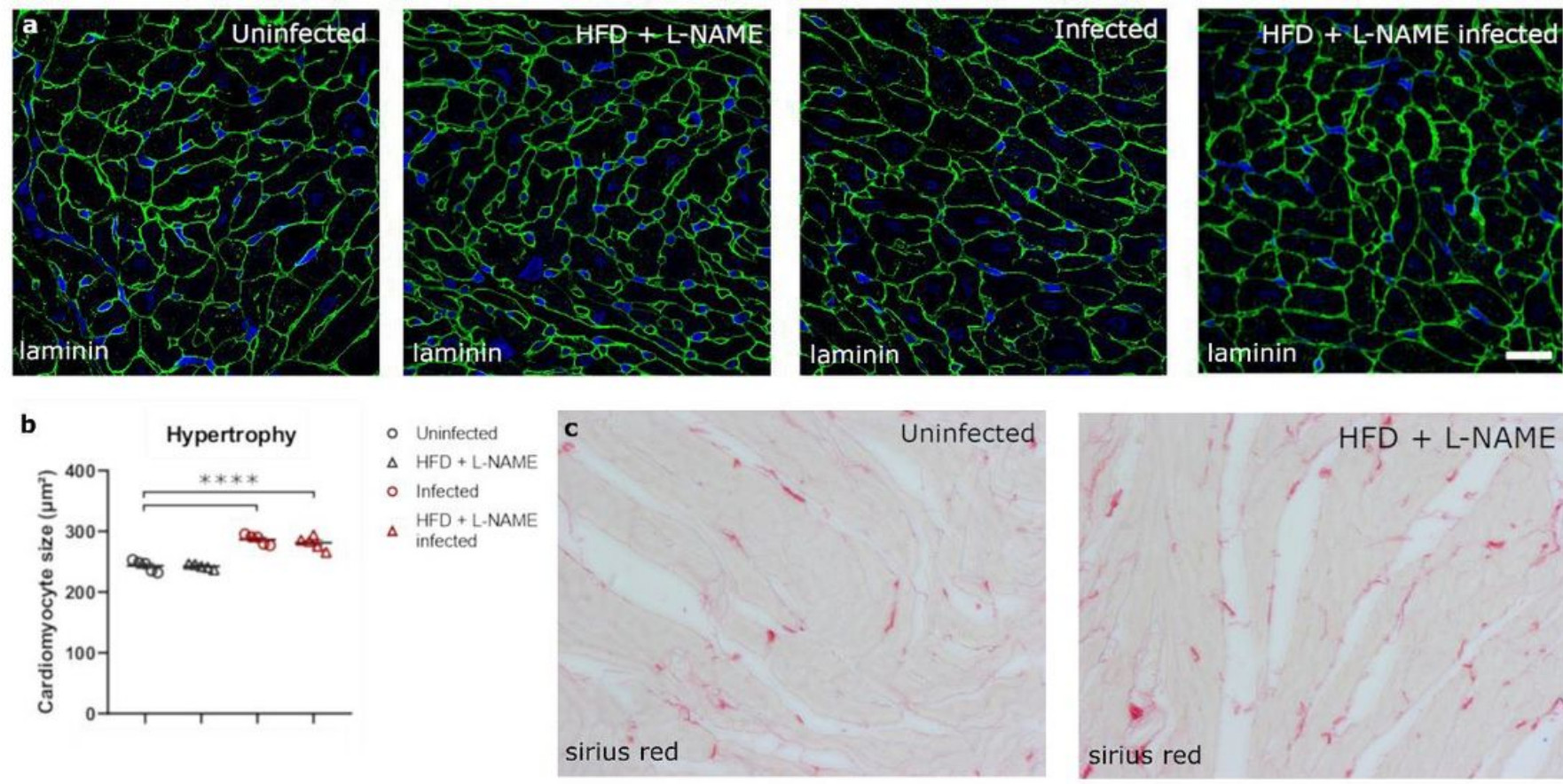

d
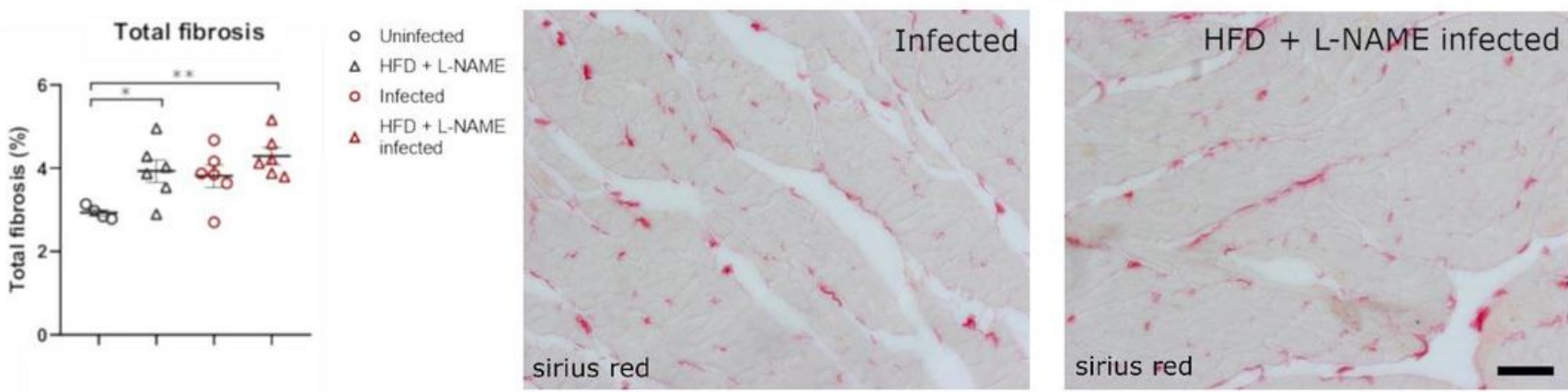

\section{Figure 4}

SARS-CoV-2 infected hamsters present with a small cardiomyocyte hypertrophy and increased cardiac fibrosis. (a) Heart sections of control and infected hamsters, either on regular chow diet or HFD + L-NAME diet, were stained for laminin and Topro3 to quantify cardiomyocyte hypertrophy (b). (c) Heart sections of control and infected hamsters, either on regular chow diet or HFD + L-NAME diet, were stained with Picro Sirius Red to analyze cardiac fibrosis (D). HFD, high fat diet; L-NAME, N y Nitro L arginine methyl ester. Values are presented as mean \pm SEM with $n=6$. Statistical analysis was analyzed using a one-way ANOVA followed by a Dunnett's multiple comparisons test, with * $p<0.05$, comparing HFD + L-NAME hamsters or infected hamsters with or without HFD + L-NAME diets to control hamsters. Scalebar represents $20 \mu \mathrm{m}(\mathrm{a}), 50 \mu \mathrm{m}$ (c). 
a

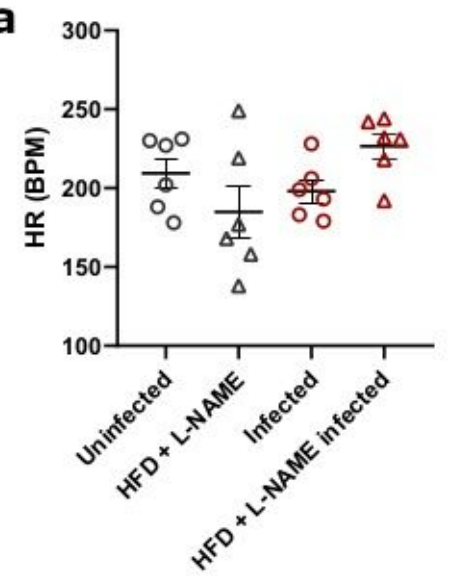

d

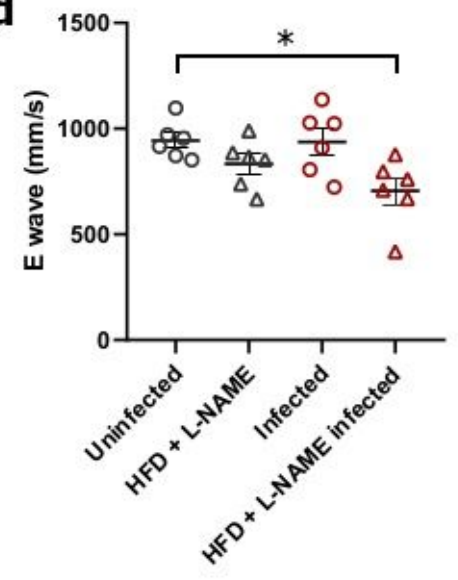

b

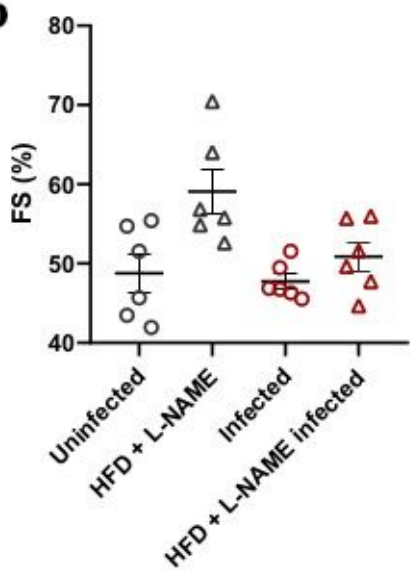

e

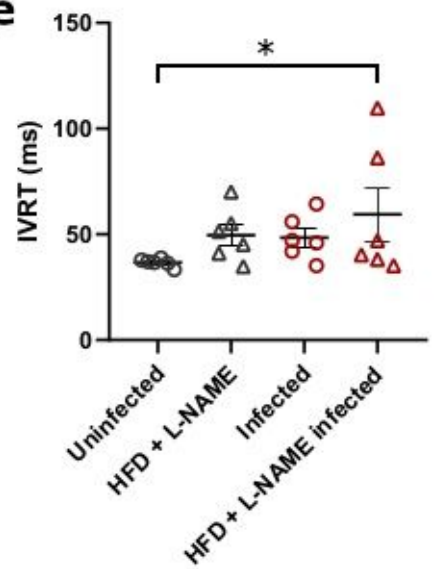

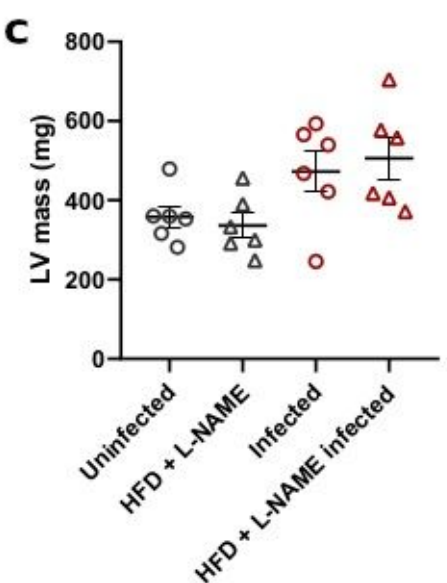

f

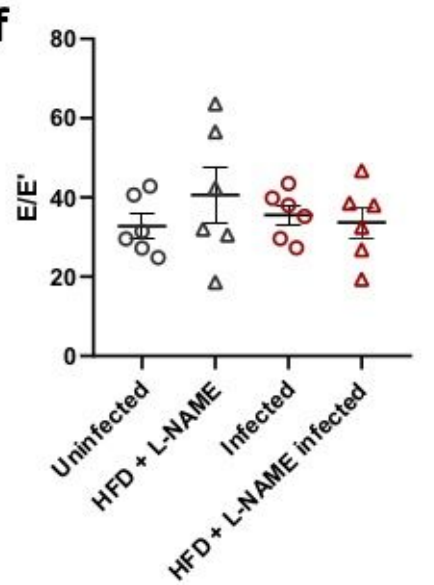

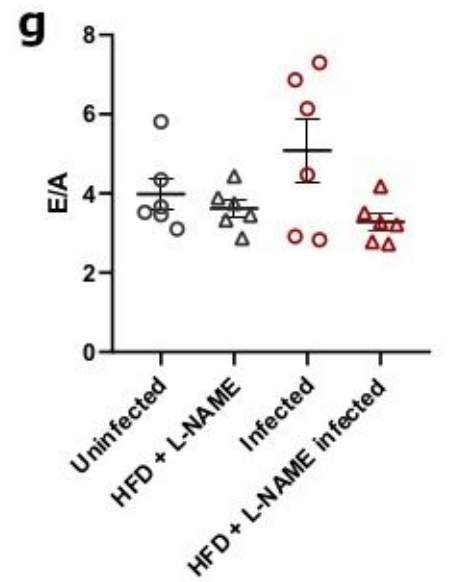

Figure 5

Echocardiography demonstrates an extremely mild diastolic dysfunction in SARS-CoV-2 infected hamsters on HFD + L-NAME diet. Cardiac function of hamsters was investigated using echocardiography two weeks after SARS-CoV-2 infection. HR (a), FS (b), E wave (c), IVRT (d), E/E' (f), and E/A (g) were analyzed. A, late mitral inflow peak velocity; E, early mitral inflow peak velocity; E', early diastolic mitral annulus peak velocity; FS, fractional shortening; HFD, high fat diet; HR, heart rate; IVRT, isovolumic relaxation time; L-NAME, $N$ y Nitro $L$ arginine methyl ester. Values are presented as mean $\pm S E M$ with $n=$ 6. All data were analyzed using a one-way ANOVA followed by a Dunnett's multiple comparison test with $* p<0.05$, ** $p<0.01$, comparing HFD + L-NAME hamsters or infected hamsters with or without HFD + LNAME diets to control hamsters. 

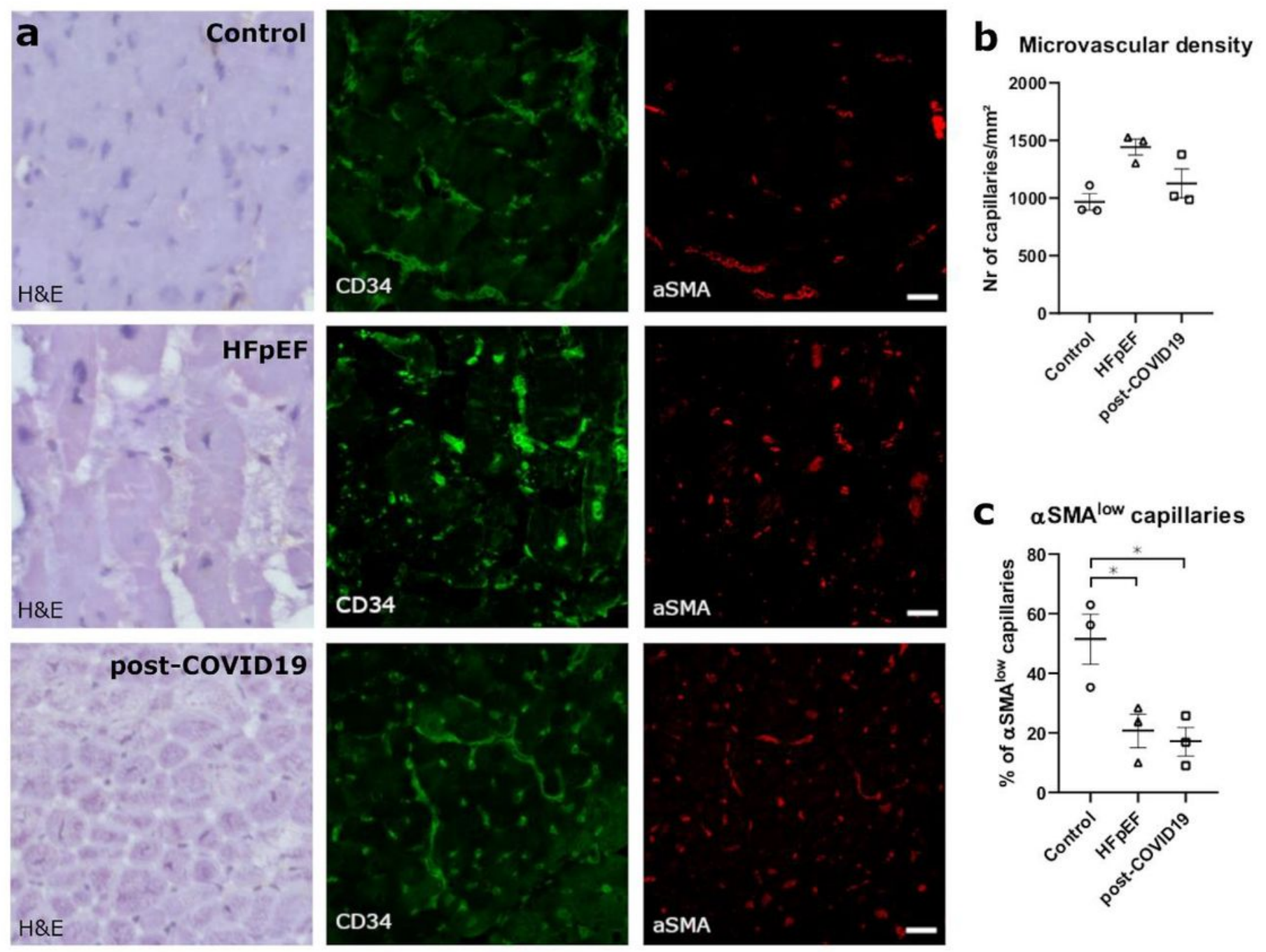

Figure 6

Recovered COVID19 patients lose the same amount of aSMAlow pericytes as patients that develop HFpEF independently of SARS-CoV-2 infection. (a) Heart biopsies from control, HFpEF patients, and recovered COVID19 patients were stained for H\&E, CD34 (green), and aSMA (red). Microvascular density (b) and the amount of aSMAlow vessels (c) were analyzed. H\&E, hematoxylin, and eosin; aSMA, alpha smooth muscle actin. Values are presented as mean \pm SEM with $n=3$ per group. Statistical analysis was performed using one-way ANOVA followed by a Dunnett's multiple comparisons test, with * $p<0.05$, comparing HFpEF patients or recovered COVID19 patients to controls. Scalebar represents $20 \mu \mathrm{m}$.

\section{Supplementary Files}

This is a list of supplementary files associated with this preprint. Click to download.

- Daemsetal.supplementary.pdf 